University of San Diego

Digital USD

$1-2016$

\title{
Structure of Sheared and Rotating Turbulence: Multiscale Statistics of Lagrangian and Eulerian Accelerations and Passive Scalar Dynamics
}

\author{
Frank G. Jacobitz \\ University of San Diego, jacobitz@sandiego.edu \\ Kai Schneider \\ University of Aix-Marseille \\ Wouter J. T. Bos \\ Ecole Centrale de Lyon \\ Marie Farge \\ Ecole Normale Superieure de Paris
}

Follow this and additional works at: https://digital.sandiego.edu/engineering_facpub

Part of the Engineering Commons, Engineering Physics Commons, and the Statistical, Nonlinear, and Soft Matter Physics Commons

\section{Digital USD Citation}

Jacobitz, Frank G.; Schneider, Kai; Bos, Wouter J. T.; and Farge, Marie, "Structure of Sheared and Rotating Turbulence: Multiscale Statistics of Lagrangian and Eulerian Accelerations and Passive Scalar Dynamics" (2016). School of Engineering: Faculty Scholarship. 29.

https://digital.sandiego.edu/engineering_facpub/29

This Article is brought to you for free and open access by the School of Engineering at Digital USD. It has been accepted for inclusion in School of Engineering: Faculty Scholarship by an authorized administrator of Digital USD. For more information, please contact digital@sandiego.edu. 


\title{
Structure of Sheared and Rotating Turbulence: Multiscale Statistics of Lagrangian and Eulerian Accelerations and Passive Scalar Dynamics
}

\begin{abstract}
The acceleration statistics of sheared and rotating homogeneous turbulence are studied using direct numerical simulation results. The statistical properties of Lagrangian and Eulerian accelerations are considered together with the influence of the rotation to shear ratio, as well as the scale dependence of their statistics. The probability density functions (pdfs) of both Lagrangian and Eulerian accelerations show a strong and similar dependence on the rotation to shear ratio. The variance and flatness of both accelerations are analyzed and the extreme values of the Eulerian acceleration are observed to be above those of the Lagrangian acceleration. For strong rotation it is observed that flatness yields values close to three, corresponding to Gaussian-like behavior, and for moderate and vanishing rotation the flatness increases. Furthermore, the Lagrangian and Eulerian accelerations are shown to be strongly correlated for strong rotation due to a reduced nonlinear term in this case. A wavelet-based scale-dependent analysis shows that the flatness of both Eulerian and Lagrangian accelerations increases as scale decreases, which provides evidence for intermittent behavior. For strong rotation the Eulerian acceleration is even more intermittent than the Lagrangian acceleration, while the opposite result is obtained for moderate rotation. Moreover, the dynamics of a passive scalar with gradient production in the direction of the mean velocity gradient is analyzed and the influence of the rotation to shear ratio is studied. Concerning the concentration of a passive scalar spread by the flow, the pdf of its Eulerian time rate of change presents higher extreme values than those of its Lagrangian time rate of change. This suggests that the Eulerian time rate of change of scalar concentration is mainly due to advection, while its Lagrangian counterpart is only due to gradient production and viscous dissipation.
\end{abstract}

\section{Keywords}

sheared turbulence, rotating turbulence, passive scalar dynamics, Lagrangian accelerations, Eulerian accelerations, rotation

\section{Disciplines}

Engineering | Engineering Physics | Statistical, Nonlinear, and Soft Matter Physics 


\title{
Structure of sheared and rotating turbulence: Multiscale statistics of Lagrangian and Eulerian accelerations and passive scalar dynamics
}

\author{
Frank G. Jacobitz, ${ }^{1, *}$ Kai Schneider, ${ }^{2, \dagger}$ Wouter J. T. Bos, ${ }^{3, \dagger}$ and Marie Farge ${ }^{4, \S}$ \\ ${ }^{1}$ Mechanical Engineering Department, Shiley-Marcos School of Engineering, University of San Diego, \\ 5998 Alcalá Park, San Diego, California 92110, USA \\ ${ }^{2}$ M2P2-CNRS and CMI, Aix-Marseille Université, 39 rue Joliot-Curie, 13453 Marseille Cedex 13, France \\ ${ }^{3}$ LMFA-CNRS, Ecole Centrale de Lyon-Université de Lyon, 36 Avenue Guy de Collongue, 69134 Ecully Cedex, France \\ ${ }^{4}$ LMD-IPSL-CNRS, Ecole Normale Supérieure, 24 rue Lhomond, 75231 Paris Cedex 5, France \\ (Received 28 July 2015; revised manuscript received 10 November 2015; published 11 January 2016)
}

\begin{abstract}
The acceleration statistics of sheared and rotating homogeneous turbulence are studied using direct numerical simulation results. The statistical properties of Lagrangian and Eulerian accelerations are considered together with the influence of the rotation to shear ratio, as well as the scale dependence of their statistics. The probability density functions (pdfs) of both Lagrangian and Eulerian accelerations show a strong and similar dependence on the rotation to shear ratio. The variance and flatness of both accelerations are analyzed and the extreme values of the Eulerian acceleration are observed to be above those of the Lagrangian acceleration. For strong rotation it is observed that flatness yields values close to three, corresponding to Gaussian-like behavior, and for moderate and vanishing rotation the flatness increases. Furthermore, the Lagrangian and Eulerian accelerations are shown to be strongly correlated for strong rotation due to a reduced nonlinear term in this case. A wavelet-based scale-dependent analysis shows that the flatness of both Eulerian and Lagrangian accelerations increases as scale decreases, which provides evidence for intermittent behavior. For strong rotation the Eulerian acceleration is even more intermittent than the Lagrangian acceleration, while the opposite result is obtained for moderate rotation. Moreover, the dynamics of a passive scalar with gradient production in the direction of the mean velocity gradient is analyzed and the influence of the rotation to shear ratio is studied. Concerning the concentration of a passive scalar spread by the flow, the pdf of its Eulerian time rate of change presents higher extreme values than those of its Lagrangian time rate of change. This suggests that the Eulerian time rate of change of scalar concentration is mainly due to advection, while its Lagrangian counterpart is only due to gradient production and viscous dissipation.
\end{abstract}

DOI: 10.1103/PhysRevE.93.013113

\section{INTRODUCTION}

The statistical description of the fluid particle acceleration is of fundamental importance for theoretical investigations of turbulence (e.g., Tsinober [1]) and for the Lagrangian modeling of particle dispersion (e.g., Pope [2]). Applications of these models include transport and mixing in geophysical flows, such as the spreading of nutrients or pollutants in the atmosphere and oceans. An initial prediction of the statistics of the fluid particle acceleration in turbulent flows can be found in the works of Heisenberg [3] and Yaglom [4]. Since then, both extensive experimental investigations, aided by the development of advanced diagnostics (e.g., La Porta et al. [5]), as well as numerous numerical simulations, enabled by the increasing power of supercomputers, have been performed. For reviews on Lagrangian properties of turbulent flows we refer the reader to Toschi and Bodenschatz [6] and Yeung [7].

The Lagrangian acceleration $d \boldsymbol{u} / d t$ consists of the two Eulerian terms $\partial \boldsymbol{u} / \partial t$ and $\boldsymbol{u} \cdot \nabla \boldsymbol{u}$, where $\boldsymbol{u}$ denotes the velocity vector in an Eulerian reference frame. By definition, the Lagrangian acceleration is invariant with respect to Galilean transformations. In contrast, the local acceleration $\partial \boldsymbol{u} / \partial t$, also

\footnotetext{
*jacobitz@sandiego.edu

${ }^{\dagger}$ kschneid@cmi.univ-mrs.fr

${ }^{\ddagger}$ wouter.bos@ec-lyon.fr

${ }^{\S}$ marie.farge@ens.fr
}

called Eulerian acceleration, depends on the reference frame. The convective acceleration $\boldsymbol{u} \cdot \boldsymbol{\nabla} \boldsymbol{u}$ contains effects due to spatial changes of the velocity vector, and it represents the nonlinearity of the Navier-Stokes equation.

Considering the incompressible Navier-Stokes equations, the Lagrangian acceleration can be further split into an irrotational part, corresponding to the pressure-gradient term $-1 / \rho_{0} \nabla p$, and a solenoidal part, corresponding to the viscous dissipation term $v \nabla^{2} \boldsymbol{u}$. For flows subjected to external body and surface forces, such as the Coriolis force or shear forces, additional terms have to be included, and such terms may alter the influence of the pressure-gradient term.

Most studies of acceleration statistics almost exclusively focus on isotropic turbulence (Toschi and Bodenschatz [6]; Yeung [7]). It was found that the Lagrangian acceleration exhibits a strong intermittency, which is reflected in the stretched-exponential tails of the probability density functions (pdfs). For example in La Porta et al. [5] it was shown that particles undergo accelerations of up to 1500 times the acceleration of gravity. Numerical simulations of isotropic turbulence confirmed these results (Toschi and Bodenschatz [6]). In addition, Bos et al. [8] analyzed and scaled the relative importance of the pressure-gradient and viscous term.

Kamps et al. [9] established a formal connection between Lagrangian and Eulerian velocity increment pdfs and gave an exact relation for the transition pdfs assuming stationarity, homogeneity, and isotropy of the flow. The limit of small 
increments yields thus the acceleration pdfs. The two transition pdfs correspond to conditional pdfs describing the transition from Eulerian pdfs to mixed Euler-Lagrangian pdfs and then to Lagrangian pdfs. In Homann et al. [10] reconstruction of Lagrangian statistics from Eulerian data using numerically calculated transition pdfs has been presented for isotropic turbulence. Modeling of the two transition pdfs has also been proposed, and good agreement with the DNS data was found.

For an incompressible Gaussian random velocity field, Holzer and Siggia [11] have shown that the pressure pdf is negatively skewed and that it has exponential tails. From that purely kinematic result it can be deduced that the pressuregradient term exhibits a Laplace distribution with flatness equal to six, as confirmed in Yoshimatsu et al. [12]. Hence, exponential tails of the Lagrangian acceleration should not be interpreted as a signature of intermittency, in contrast to stretched exponential tails. This has been discussed in Bos et al. [13] in the context of drift-wave turbulence.

Scale-dependent statistics for turbulent flows using the orthogonal wavelet decomposition have been introduced in Meneveau [14], Farge [15], and more recently Bos et al. [16] and have been applied to study acceleration statistics for isotropic turbulence in Yoshimatsu et al. [12]. There it was shown that intermittency increases at smaller scales. The influence of nonslip walls on the Lagrangian acceleration has been investigated in Kadoch et al. [17]. It was found that the boundary layer which generates vorticity leads to even stronger intermittency of the acceleration compared to homogeneous flows.

The aim of the present work is to study acceleration statistics in turbulent shear flow. We consider three types of homogeneous shear flows: without rotation, with moderate rotation, and with strong rotation, where the direction of system rotation is either parallel or antiparallel to the dominant vorticity component induced by the mean shear. The direct numerical simulation data discussed in Jacobitz et al. [18,19] are analyzed to study the statistics of Lagrangian and Eulerian accelerations with a particular focus on the influence of shear and rotation as well as on the scale dependence of the statistics. In addition, the variances of the Lagrangian and Eulerian accelerations are compared to estimates obtained by scaling arguments applied to the terms in the equations of motion.

Moreover, the time rate of change of passive scalar fluctuations is studied from Lagrangian and Eulerian points of view. The passive scalar can represent a variety of quantities, including pollutants or nutrients transported in geophysical flows. An additional aim of the present study is to analyze the influence of rotation on the structure and dynamics of a passive scalar. A similar flow configuration has been studied by Brethouwer [20] using a much higher shear rate in order to enable a comparison with boundary layer turbulence. Brethouwer observed a strong dependence of the scalar flux on the rotation to shear ratio and an agreement with predictions from linear theory in the high shear range.

The outline of the paper is the following. In Sec. II, the equations of motion are recalled, the numerical simulations are described, and estimates for the variances of the Lagrangian and Eulerian accelerations are obtained. The results of the acceleration statistics, including a detailed assessment of the different contributions and a scale dependent analysis, are presented in Sec. III, together with some passive scalar statistics. Finally, in Sec. IV conclusions are drawn. Appendix A summarizes the acceleration statistics for an isotropic turbulence field.

\section{MATHEMATICAL PRELIMINARIES AND PHENOMENOLOGICAL ESTIMATES}

In this section, the equations of motion are introduced, the numerical approach is discussed, and an estimation of the variances of the Lagrangian and Eulerian accelerations is provided.

\section{A. Equations of motion}

This study is based on existing direct numerical simulation results of sheared and rotating homogeneous turbulence $[18,19]$. Properties of homogeneous turbulence with shear and rotation are discussed in Ref. [21], and a general discussion of homogeneous turbulence can be found in the monograph of Sagaut and Cambon [22].

A decomposition of the total velocity into a constant mean part $\boldsymbol{U}=(U, V, W)$ with

$$
U=S y, \quad V=W=0
$$

leads to the following form of the incompressible NavierStokes equations for the fluctuating velocity $\boldsymbol{u}=(u, v, w)$ :

$$
\begin{gathered}
\nabla \cdot \boldsymbol{u}=0, \\
\frac{\partial \boldsymbol{u}}{\partial t}+\boldsymbol{u} \cdot \nabla \boldsymbol{u}+S y \frac{\partial \boldsymbol{u}}{\partial x}+S v \boldsymbol{e}_{x}+2 \boldsymbol{\Omega} \times \boldsymbol{u} \\
=-\frac{1}{\rho_{0}} \nabla p+v \nabla^{2} \boldsymbol{u} .
\end{gathered}
$$

Here $S=\partial U / \partial y$ is the constant shear rate, $f=2 \Omega$ the constant Coriolis parameter for system rotation about the $z$-coordinate axis, $\rho_{0}$ the density, $v$ the kinematic viscosity, and $\boldsymbol{e}_{x}$ the unit vector in the $x$ direction.

Similarly, a decomposition of a passive scalar into a constant mean part $C$ with

$$
C=S_{c} y
$$

leads to the following advection-diffusion equation for the fluctuating scalar component $c$ :

$$
\frac{\partial c}{\partial t}+\boldsymbol{u} \cdot \nabla c+S y \frac{\partial c}{\partial x}+S_{c} v=\alpha \nabla^{2} c .
$$

In this equation, $S_{c}=\partial C / \partial y$ is the constant scalar gradient in the $y$ direction and $\alpha$ is the molecular diffusion rate of the scalar. A schematic of the mean flow is shown in Fig. 1.

The above equations are transformed into a frame of reference moving with the $y$-dependent mean velocity by applying the Rogallo transform [23], which is also called shearing box approximation in the astrophysics community. In the Rogallo frame, the equations of motion for fluctuating velocity and scalar read:

$$
\boldsymbol{\nabla} \cdot \boldsymbol{u}-\mathrm{St} \frac{\partial v}{\partial x}=0
$$




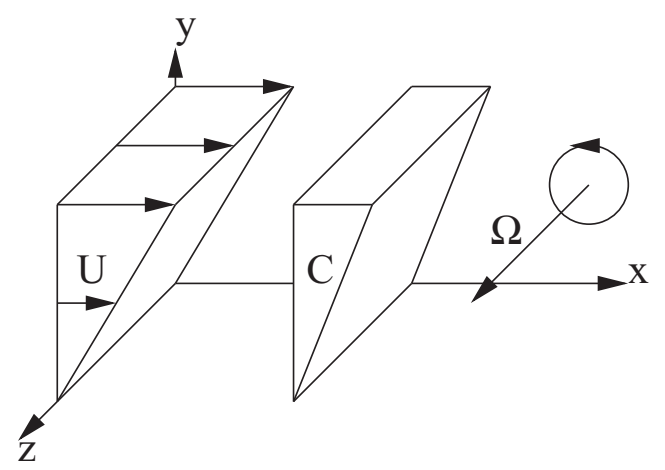

FIG. 1. Schematic of the mean flow with mean velocity $U$, mean scalar $C$, and system rotation $\Omega$. In this example the system rotation is antiparallel to the vorticity from the mean shear.

$$
\begin{aligned}
\frac{\partial \boldsymbol{u}}{\partial t}+\boldsymbol{u} \cdot \nabla \boldsymbol{u}-\mathrm{St} w \frac{\partial \boldsymbol{u}}{\partial x}+S_{v} \boldsymbol{e}_{x}+2 \boldsymbol{\Omega} \times \boldsymbol{u} \\
=-\frac{1}{\rho_{0}}\left(\nabla p-\mathrm{St} \frac{\partial p}{\partial x} \boldsymbol{e}_{y}\right) \\
+v\left[\nabla^{2}-2 \mathrm{St} \frac{\partial^{2}}{\partial x \partial y}+(\mathrm{St})^{2} \frac{\partial^{2}}{\partial^{2} x}\right] \boldsymbol{u}, \\
\frac{\partial c}{\partial t}+\boldsymbol{u} \cdot \nabla c-\mathrm{St} v \frac{\partial c}{\partial x}+S_{c} v \\
=\alpha\left[\nabla^{2}-2 \mathrm{St} \frac{\partial^{2}}{\partial x \partial y}+(\mathrm{St})^{2} \frac{\partial^{2}}{\partial^{2} x}\right] c .
\end{aligned}
$$

In addition, a regridding approach is used: when the simulations reach the nondimensional time $(=$ shear rate $\times$ time) $\mathrm{St}=0.5$, the grid is remapped to a nondimensional time $\mathrm{St}=-0.5$. The regridding is accomplished using the periodic properties of the turbulent fields, and it does not require any interpolation at the chosen nondimensional time. Hence, the local time St in the Rogallo frame with regridding varies repeatedly from $\mathrm{St}=-0.5$ to 0.5 and additional regridding procedures are applied when $\mathrm{St}=0.5$ is repeatedly reached. The Rogallo frame is not distorted for $\mathrm{St}=0$, corresponding to integer values of the nondimensional time, and the flow is analyzed at those integer times for simplicity. Jacobitz et al. [24] provide a more detailed explanation of the Rogallo transform and the regridding approach.

\section{B. Numerical simulations}

In a flow with uniform mean gradients, the use of the Rogallo reference frame enables the application of periodic boundary conditions for the fluctuating components of velocity and for the passive scalar. A spectral collocation method is used for the spatial discretization. The solution is advanced in time with a fourth-order Runge-Kutta scheme. The simulations are performed on a parallel computer using $256 \times 256 \times 256$ grid points. More details about the simulations used in this study can be found in Jacobitz et al. [18,19].

The results from five simulations of sheared and rotating turbulence with rotation to shear ratios of $f / S=-5,-0.5$, $0,+0.5$, and +5 are considered. Negative values of $f / S$ correspond to a parallel configuration and positive values correspond to an antiparallel configuration between the system rotation and the mean flow vorticity. Isotropic turbulence fields are used to initialize all simulations and the initial values of the Taylor microscale Reynolds number $\operatorname{Re}_{\lambda}=q \lambda / \nu=45$ and the shear number $S K / \epsilon=2$ are fixed, where $K$ denotes the turbulent kinetic energy, $q=\sqrt{2 K}$ the velocity magnitude, $\lambda=5 v q^{2} / \epsilon$ the Taylor microscale, and $\epsilon$ the dissipation rate.

The initial value of the Taylor microscale Reynolds number is chosen to fully resolve all dynamically important scales throughout the simulations. Its values depend on the eventual evolution of the turbulence and reach values as high as $\operatorname{Re}_{\lambda}=$ 120 . The shear number varies only weakly with $f / S$, and it eventually assumes a value of about $S K / \epsilon=6$. In comparison to Brethouwer [20], who uses a high shear rate to enable a comparison to boundary layer turbulence, the shear rate in the present study is moderate and results in a fully nonlinear evolution of the base shear flow without rotation. Hence, the impact of a variation of the rotation to shear ratio $f / S$ on the flow dynamics can be studied. The initial energy spectrum has a peak at wave number $k=10$, which allows for both largeand small-scale growth as the simulations advance in time. The simulations are initialized without scalar fluctuations, and the Prandtl number is $\operatorname{Pr}=v / \alpha=0.7$.

The eventual evolution of the flow, growth or decay, depends on the value of the rotation to shear ratio $f / S$. The nonrotating case with $f / S=0$ shows eventual exponential growth of the kinetic energy $K$. For moderate rotation to shear ratios, the antiparallel case with $f / S=+0.5$ leads to a strong growth of the turbulent kinetic energy, while the parallel case with $f / S=-0.5$ results in a decay of $K$. For strong rotation to shear ratios, however, both the antiparallel case with $f / S=+5$ and the parallel case with $f / S=-5$ lead to a strong decay of $K$ due to the importance and dominance of linear effects.

\section{Lagrangian and Eulerian accelerations}

In this section, the Lagrangian and Eulerian accelerations are introduced, and estimates for their variances are obtained for isotropic turbulence as well as sheared and rotating turbulence. Finally, the Lagrangian and Eulerian time rates of change for a scalar are introduced as well.

\section{Expressions for the accelerations}

The Lagrangian and Eulerian accelerations are defined as

$$
\boldsymbol{a}_{L}=\frac{\partial \boldsymbol{u}}{\partial t}+\boldsymbol{u} \cdot \nabla \boldsymbol{u}
$$

and

$$
\boldsymbol{a}_{E}=\frac{\partial \boldsymbol{u}}{\partial t}
$$

respectively. The Lagrangian and Eulerian accelerations are computed as a volume average at a fixed time. The volume average is an appropriate choice for homogeneous flow and effects of shear and rotation are considered to be external forces.

The Navier-Stokes equation can be written as

$$
\frac{\partial \boldsymbol{u}}{\partial t}=-\boldsymbol{N}-\boldsymbol{\Pi}-\boldsymbol{\Lambda},
$$


and at integer nondimensional time St the right-hand-side terms take the form

$$
\begin{aligned}
\boldsymbol{N} & =\boldsymbol{u} \cdot \nabla \boldsymbol{u}, \quad \boldsymbol{\Pi}=\nabla\left(p / \rho_{0}\right), \\
\boldsymbol{\Lambda} & =S v \boldsymbol{e}_{x}+2 \boldsymbol{\Omega} \times \boldsymbol{u}-v \nabla^{2} \boldsymbol{u} .
\end{aligned}
$$

Here $\boldsymbol{N}$ is the nonlinear term, $\boldsymbol{\Pi}$ the pressure-gradient term, and $\boldsymbol{\Lambda}$ the linear term with contributions from shear, rotation, and viscous effects.

Taking the divergence of the Navier-Stokes equation (11), a Poisson equation for the pressure is obtained,

$$
\nabla^{2} p / \rho_{0}=-\nabla \cdot(\boldsymbol{N}+\boldsymbol{\Lambda})
$$

and we prove the identity

$$
\left\langle\|\boldsymbol{N}+\boldsymbol{\Pi}+\boldsymbol{\Lambda}\|^{2}\right\rangle=\left\langle\|\boldsymbol{N}+\boldsymbol{\Lambda}\|^{2}\right\rangle-\left\langle\|\boldsymbol{\Pi}\|^{2}\right\rangle
$$

in Appendix B. Here $\|\cdot\|$ denotes the magnitude of a vector and $\langle\cdot\rangle$ the volume average for a homogeneous field.

Hence, the variance of the Eulerian acceleration is given by

$$
\begin{aligned}
a_{E}^{2} \equiv\left\langle\left\|\frac{\partial \boldsymbol{u}}{\partial t}\right\|^{2}\right\rangle=\left\langle\|\boldsymbol{N}+\boldsymbol{\Pi}+\boldsymbol{\Lambda}\|^{2}\right\rangle \\
=\left\langle\|\boldsymbol{N}+\boldsymbol{\Lambda}\|^{2}\right\rangle-\left\langle\|\boldsymbol{\Pi}\|^{2}\right\rangle,
\end{aligned}
$$

and the variance of the Lagrangian acceleration is obtained as

$$
a_{L}^{2} \equiv\left\langle\left\|\frac{\partial \boldsymbol{u}}{\partial t}+\boldsymbol{u} \cdot \nabla \boldsymbol{u}\right\|^{2}\right\rangle=\left\langle\|\boldsymbol{\Pi}+\boldsymbol{\Lambda}\|^{2}\right\rangle .
$$

The goal now is to give an estimate of the different contributions to the acceleration as a function of dimensionless quantities.

\section{Isotropic turbulence}

For isotropic turbulence the shear and rotation terms are zero and the expression for the linear term becomes $\boldsymbol{\Lambda}=$ $-v \nabla^{2} \boldsymbol{u}$. Taking the divergence of the Navier-Stokes equations, we obtain a Poisson equation for the pressure

$$
\nabla^{2} p / \rho_{0}=-\nabla \cdot N \text {. }
$$

The pressure is thus determined by the divergence of the nonlinear term alone. Since this term is a quadratic quantity, even in the case of a Gaussian velocity field, the pressure will be non-Gaussian due to this nonlinearity [11].

We now introduce the notation

$$
N^{2}=\left\langle\|\boldsymbol{N}\|^{2}\right\rangle
$$

for the variance of the nonlinear term and

$$
\Pi_{N L}^{2}=\left\langle\|\Pi\|^{2}\right\rangle_{\text {Isotropic }}
$$

for the variance of the pressure gradient. The subscript $N L$ indicates the fact that in isotropic turbulence the pressure is entirely determined by the nonlinear interaction term.

For turbulent flows, the viscous contribution to the acceleration is small [25], and it will be neglected in the following. The expressions for the variance of the Eulerian and Lagrangian acceleration become then, respectively,

$$
\begin{gathered}
a_{E}^{2} \approx N^{2}-\Pi_{N L}^{2}, \\
a_{L}^{2} \approx \Pi_{N L}^{2} .
\end{gathered}
$$

In isotropic turbulence at high Reynolds number, the magnitude of the nonlinear term can be estimated using a Tennekes sweeping estimate $[8,26]$ as

$$
N^{2} \sim U^{2} \epsilon / \nu \sim \frac{U^{4}}{L^{2}} R_{L},
$$

where

$$
R_{L}=U L / v
$$

is an integral-scale Reynolds number. An appropriate choice for the velocity scale is the magnitude of the fluctuating velocity $U=q$ and the integral scale can be estimated as $L \sim U^{3} / \epsilon$. This scaling follows from the assumption that the velocity gradients, measured by $\epsilon / \nu$, become independent of the large-scale velocities, measured by $U$. This approximation seems to hold already at relatively low Reynolds numbers [8].

The pressure-gradient approximately satisfies Kolmogorov scaling [27] leading to

$$
\Pi_{N L}^{2} \sim \epsilon^{3 / 2} / \nu^{1 / 2} \sim \frac{U^{4}}{L^{2}} R_{L}^{1 / 2}
$$

where the second approximation again uses $L \sim U^{3} / \epsilon$. Therefore, for isotropic turbulence at large Reynolds numbers, the variance of the Eulerian acceleration is large compared to the variance of the Lagrangian acceleration:

$$
a_{E}^{2} \gg a_{L}^{2}
$$

\section{Rotating shear flow}

We will now determine the order of magnitude of the linear terms in sheared and rotating turbulence. To obtain an estimate, we will consider the turbulence to be isotropic. This approximation is thus valid only at the beginning of the simulations. At later times deviations from the following results are expected as the isotropy assumption is a crucial simplification. The validity of this assumption can only be assessed a posteriori. However, strong assumptions are needed if anything sensible is to be derived from the equations without introducing too complicated technical machinery. Ignoring the viscous contribution, we find from (12) that

$$
\Lambda^{2} \equiv\left\langle\|\mathbf{\Lambda}\|^{2}\right\rangle=\frac{1}{3} S^{2}\left(1-2 \frac{f}{S}+2 \frac{f^{2}}{S^{2}}\right)\left\langle\|\boldsymbol{u}\|^{2}\right\rangle .
$$

In the case of rotating shear flow, the Poisson equation now contains the linear contribution as in Eq. (13). In order to simplify the considerations, we will solve the equation

$$
\nabla^{2} p / \rho_{0}=-\nabla \cdot \boldsymbol{\Lambda}
$$

to obtain an estimate for the strength of the linear contribution to the pressure gradient $\Pi_{L}$. This amounts to a rapid distortion approximation [28] valid in the limit of strong linear effects. This is a straightforward but rather tedious computation, which we carried out in Fourier space. We find that

$$
\Pi_{L}^{2} \equiv\left\langle\left\|\boldsymbol{\Pi}_{L}\right\|^{2}\right\rangle=\frac{2}{15} S^{2}\left(1-\frac{5}{2} \frac{f}{S}+\frac{5}{2} \frac{f^{2}}{S^{2}}\right)\left\langle\|\boldsymbol{u}\|^{2}\right\rangle .
$$


We now consider order of magnitude estimates in the limit of strong and weak linear effects, using the rotation number

$$
\frac{f}{S}
$$

and the shear number

$$
\frac{S K}{\epsilon},
$$

where $K$ is the kinetic energy.

In the limit of strong rotation we find

$$
\Pi_{L}^{2} \sim \frac{U^{4}}{L^{2}}\left(\frac{f}{S}\right)^{2}, \quad \Lambda^{2} \sim \frac{U^{4}}{L^{2}}\left(\frac{f}{S}\right)^{2} .
$$

Equivalently, for strong shear, we obtain

$$
\Pi_{L}^{2} \sim \frac{U^{4}}{L^{2}}\left(\frac{S K}{\epsilon}\right)^{2}, \quad \Lambda^{2} \sim \frac{U^{4}}{L^{2}}\left(\frac{S K}{\epsilon}\right)^{2} .
$$

If the linear effects are weak, the dominant terms are

$$
\Pi_{N L}^{2} \sim \frac{U^{4}}{L^{2}} R_{L}^{1 / 2}, \quad N^{2} \sim \frac{U^{4}}{L^{2}} R_{L} .
$$

For pure shear flow to dominate the Lagrangian acceleration, we need thus that the linear pressure contribution becomes stronger than the nonlinear contribution, implying

$$
\left(\frac{S K}{\epsilon}\right)^{2} \gg R_{L}^{1 / 2} .
$$

For the linear effects to dominate the Eulerian acceleration, we need the stronger condition

$$
\left(\frac{S K}{\epsilon}\right)^{2} \gg R_{L}
$$

since the norm of the nonlinear term is determined by the sweeping of the large scales. Similar relations, replacing $S K / \epsilon$ by $f / S$ will give the conditions for rotation to be the dominant contribution to the Lagrangian acceleration as

$$
\left(\frac{f}{S}\right)^{2} \gg R_{L}^{1 / 2}
$$

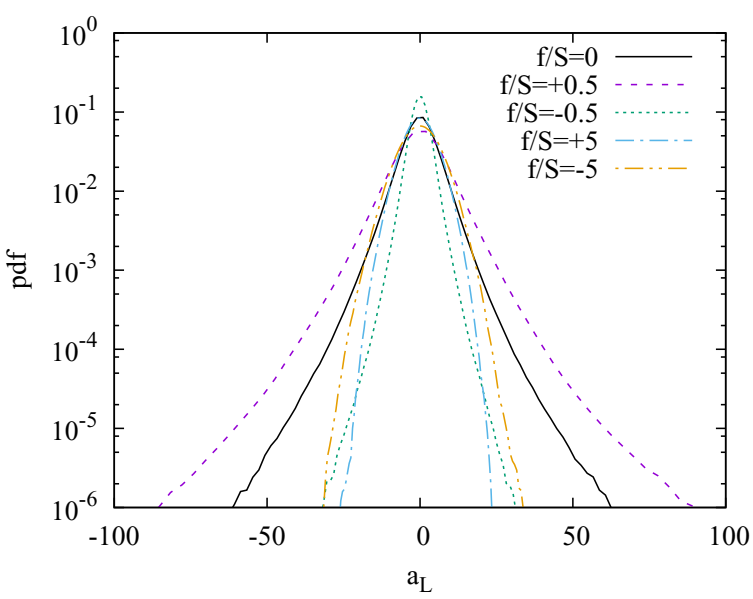

and for the Eulerian acceleration as

$$
\left(\frac{f}{S}\right)^{2} \gg R_{L} \text {. }
$$

\section{Scalar rate of change}

Similarly to the accelerations, the time rate of change of the scalar fluctuations can be considered from Lagrangian and Eulerian point of views:

$$
s_{L}=\frac{\partial c}{\partial t}+\boldsymbol{u} \cdot \nabla c
$$

and

$$
s_{E}=\frac{\partial c}{\partial t}
$$

respectively.

The scalar equation can be written as

$$
\frac{\partial c}{\partial t}=-N_{c}-\Lambda_{c}
$$

where the term $N_{c}$ for the advection of scalar concentration and the term $\Lambda_{c}$ for the diffusion of scalar concentration take the following forms for integer nondimensional time St:

$$
N_{c}=\boldsymbol{u} \cdot \nabla c, \quad \Lambda_{c}=S_{c} v-\alpha \nabla^{2} c .
$$

We will now focus on the scaling of $N_{c}^{2}$ and $\Lambda_{c}^{2}$. When advecting a passive scalar in isotropic turbulence, the term $N_{c}^{2}$ can be estimated by a sweeping estimate [29] such as

$$
N_{c}^{2} \sim U^{2} \epsilon_{c} / \alpha
$$

where $\epsilon_{c}$ is the scalar dissipation rate. In the presence of a uniform scalar gradient, the production term will be of order of magnitude (assuming isotropy $\left\langle v^{2}\right\rangle=U^{2} / 3$ )

$$
O\left(S_{c} v\right) \sim U S_{c}
$$

Estimating that the dissipation of scalar fluctuations is in approximate balance with the production term, we have $\epsilon_{c} \sim S_{c}^{2} L U$. We therefore obtain the following estimates for the advection and linear terms of the scalar equation:

$$
N_{c}^{2} \sim\left(U S_{c}\right)^{2} \frac{U L}{\alpha}
$$

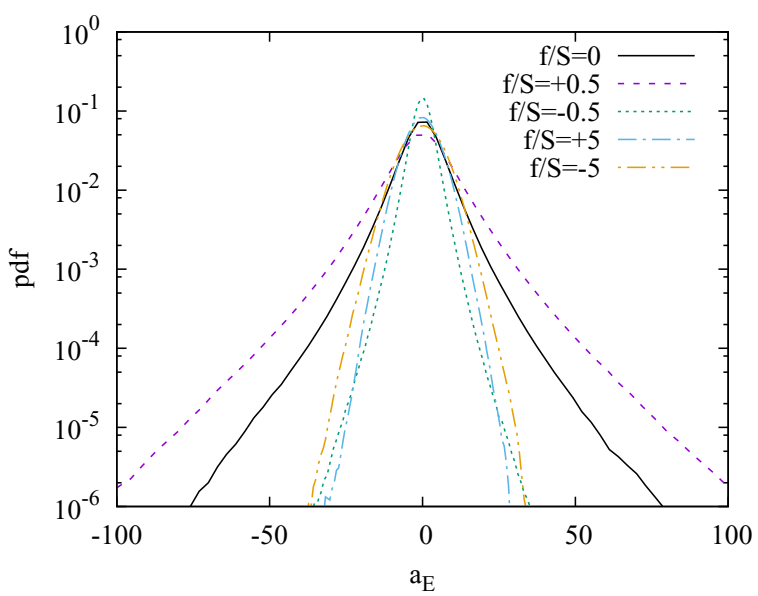

FIG. 2. Pdfs of Lagrangian (left) and Eulerian (right) accelerations. 

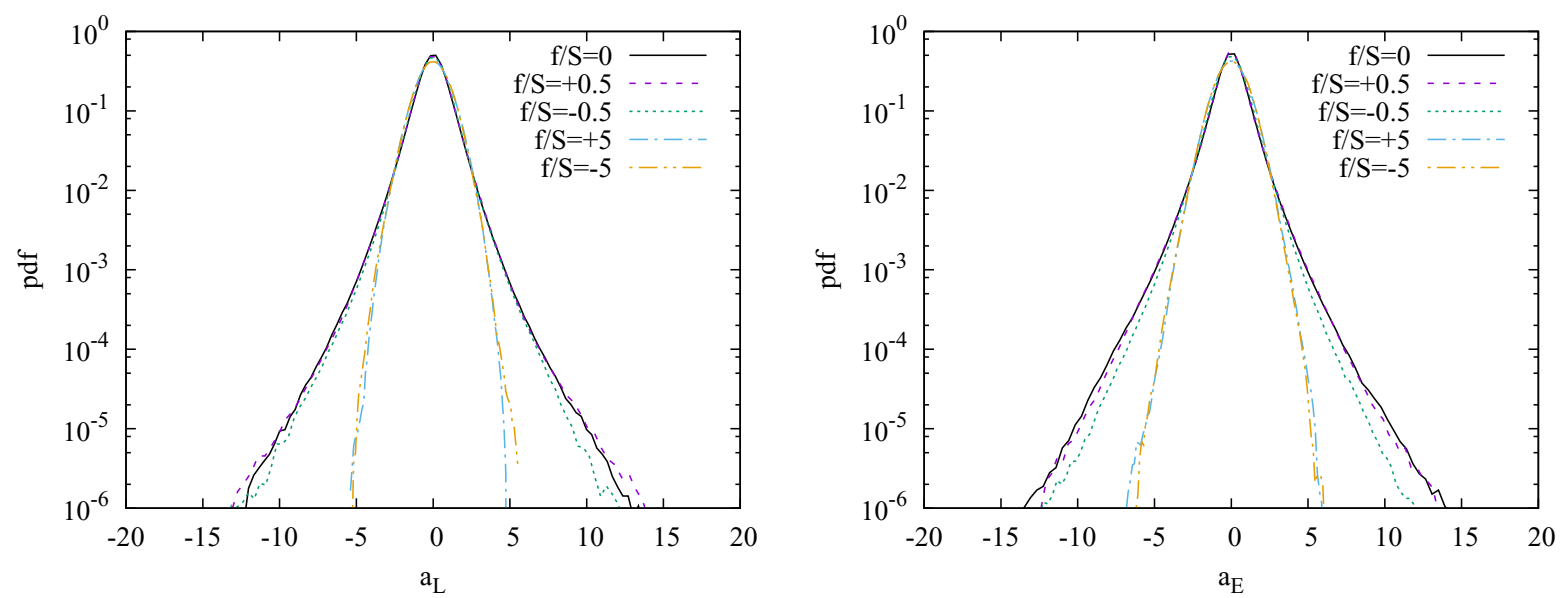

FIG. 3. Pdfs normalized with their corresponding standard deviation of Lagrangian (left) and Eulerian (right) accelerations.

and

$$
\Lambda_{c}^{2} \sim\left(U S_{c}\right)^{2}
$$

The mean-square advection term (and Eulerian rate of change) is hence dominant over the linear term (and Lagrangian rate of change) at high Péclet number Pe, which is the product of Reynolds number and Prandtl number Pr $=\mathrm{Pe} /$ Re. Since rotation and shear do not appear explicitly in the scalar evolution equation, we cannot provide any further prediction considering those equations only.

\section{RESULTS}

In this section, the statistical properties of Lagrangian and Eulerian accelerations are discussed, the contributions to those accelerations from the different terms of the Navier-Stokes equation to the accelerations are presented, the scale dependence of the accelerations is considered, and the Lagrangian and Eulerian time rates of change of passive scalar fluctuations are investigated. The results are presented at nondimensional time $\mathrm{St}=5$. At that nondimensional time, the effects of shear are developed and all cases, including those with strongly growing turbulent kinetic energy, are well resolved at the largest and smallest scales of the motion.

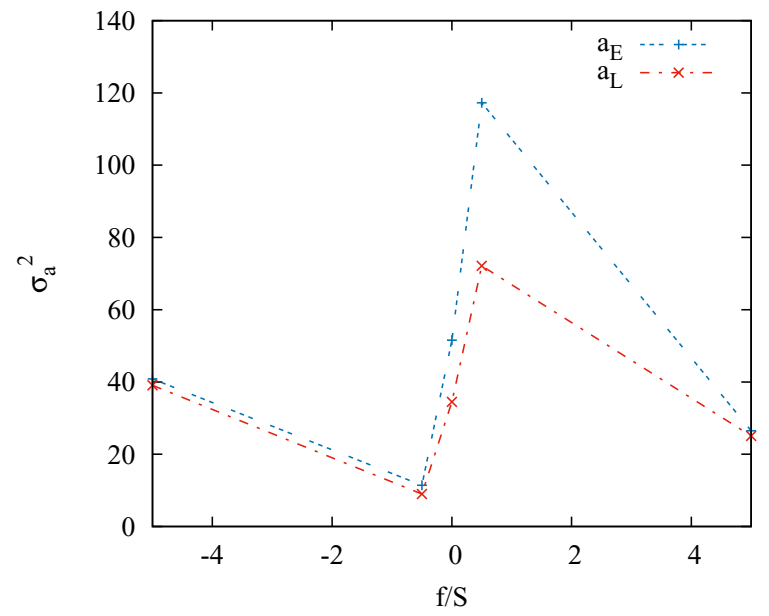

\section{A. Acceleration statistics}

The pdfs (one-point pdfs estimated using histograms with 128 bins and averaging over the three components) of Lagrangian and Eulerian accelerations for sheared and rotating turbulence at nondimensional time $\mathrm{St}=5$ are shown in Fig. 2. A strong and similar dependence on the rotation to shear ratio $f / S$ is obtained for both accelerations. Figure 3 presents both acceleration pdfs normalized with their corresponding standard deviation. Two families of pdfs are obtained: Gaussian-like behavior for strong rotation with $f / S= \pm 5$ and stretched-exponential-like behavior for the remaining cases.

Figure 4 (left) compares the variances of the Lagrangian and Eulerian accelerations. The variance of the Eulerian acceleration $a_{E}$ is always larger than the variance of the Lagrangian acceleration $a_{L}$ at a given value of $f / S$ in agreement with results for isotropic turbulence (see Appendix A). Also, the extreme values of the Eulerian acceleration are above those of the Lagrangian acceleration for all cases in Fig. 2. The fluctuating pressure gradients thus seem to be less important in rotating and sheared turbulence as compared to isotropic turbulence. The difference is most pronounced for the case with $f / S=+0.5$, while the variance values are almost equal for the strongly rotating cases with $f / S= \pm 5$.

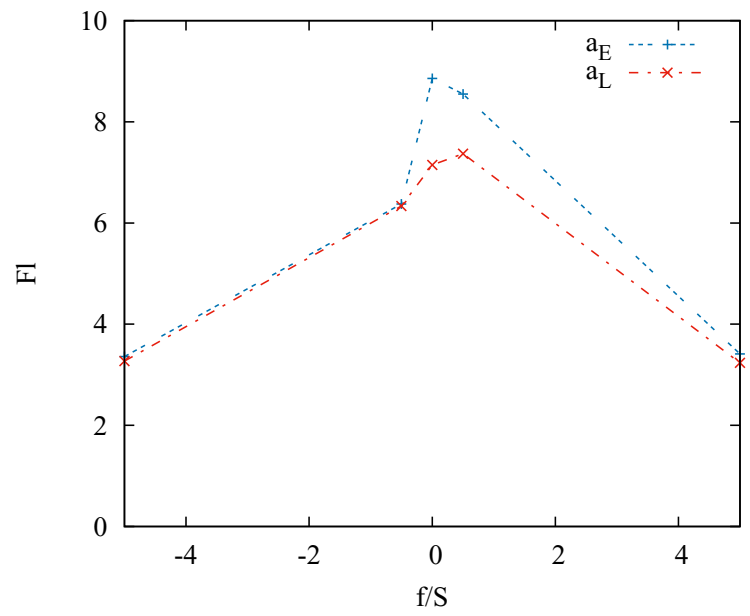

FIG. 4. Variance (left) and flatness (right) of Lagrangian and Eulerian accelerations as a function of $f / S$. 

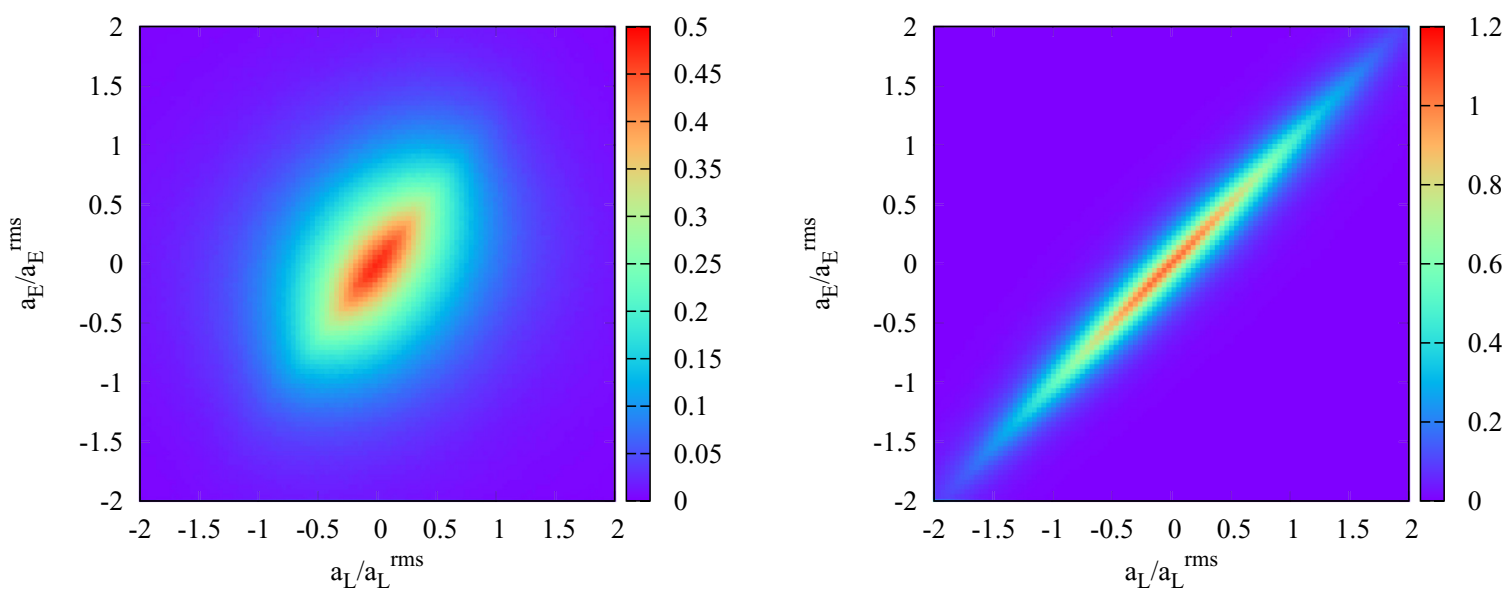

FIG. 5. Joint pdfs of Lagrangian and Eulerian accelerations for $f / S=+0.5$ (left) and $f / S=+5$ (right).

The variances of both accelerations are smallest for the case with $f / S=-0.5$. This is due to the pressure-gradient term to be discussed in the next section.

Contrary to the results for isotropic turbulence reported by Ishihara et al. [30] (see also Appendix A), the Eulerian acceleration pdfs consistently exhibit heavier tails than their Lagrangian counterparts at the Reynolds number of this study. This behavior is also reflected in the flatness, which is defined as the ratio of the fourth order moment divided by the square of the second order moment, which is shown in Fig. 4 (right). The flatness can also be used to quantify the influence of the rotation to shear ratio $f / S$ on the intermittency of the turbulent flow. For strong rotation with $f / S= \pm 5$, we find flatness values close to three, which confirms the Gaussian-like behavior of the acceleration pdfs. For the remaining cases larger values are observed for the flatness, with a maximum at $f / S=0$ for the Eulerian acceleration, and $f / S=+0.5$ for the Lagrangian acceleration. For positive values of $f / S$, the flatness of the Eulerian acceleration is larger than the flatness of the Lagrangian acceleration. The difference between the two accelerations decreases with increasing $f / S$, and for $f / S=$ +5 we find a value close to three.

To check the correlation between Lagrangian and Eulerian accelerations, we show in Fig. 5 the joint pdfs for a strongly growing case with $f / S=+0.5$ and a decaying case with $f / S=+5$. The results indicate that the case with strong rotation with $f / S=+5$ exhibits indeed a strong correlation. To further quantify the level of correlation, the Pearson product-moment correlation coefficient $r$ ([[31], p. 162]) is given in Table I for a computation based on the three

TABLE I. Pearson product-moment correlation coefficient $r$ for the Lagrangian and Eulerian accelerations at nondimensional time $\mathrm{St}=5$. The correlation coefficient is determined for all three components as well as the magnitude of the accelerations.

\begin{tabular}{lrrrrr}
\hline \hline$f / S$ & -5 & -0.5 & 0 & +0.5 & +5 \\
\hline$r$ (component) & 0.9620 & 0.2825 & 0.0926 & 0.0826 & 0.9507 \\
$r$ (magnitude) & 0.9186 & 0.3267 & 0.3175 & 0.2822 & 0.9010 \\
\hline \hline
\end{tabular}

acceleration components as well as for a computation based on the acceleration magnitudes.

For a computation of the Pearson product-moment correlation coefficient $r$ based on the three components of Lagrangian and Eulerian accelerations, small values are found for the cases with growing turbulence with $f / S=0$ and $f / S=+0.5$, which indicates a lack of correlation between the accelerations. A moderate value is obtained for the slightly decaying case with $f / S=-0.5$, and a large value is observed for the strongly rotating cases with $f / S= \pm 5$, indicating a strong correlation for the Lagrangian and Eulerian accelerations. This large correlation coefficient is due to a reduced nonlinear term in the cases with strong rotation. For a computation of the Pearson product-moment correlation coefficient $r$ based on the magnitudes of the Lagrangian and Eulerian accelerations, a moderate level of correlation is observed for the cases with growing turbulence with $f / S=0$ and $f / S=+0.5$.

The anisotropy of the Lagrangian and Eulerian accelerations is considered in Fig. 6 and Table II. The normalized Lagrangian and Eulerian acceleration pdfs do not differ significantly in shape between the vector fields and the different vector components as shown in Fig. 6. Hence, the anisotropy of the accelerations is described by their variances, which are provided in Table II. We observe that the anisotropy is most pronounced for the strongly rotating cases with $f / S= \pm 5$. The variance of the $z$ components is reduced by almost a factor of two.

\section{B. Contributions to the acceleration statistics}

To clarify our understanding of the Lagrangian and Eulerian acceleration pdfs, we now consider the pdfs of the velocity and of all terms in the Navier-Stokes equation, as shown in Fig. 7. To quantify the relative importance and shapes of the pdfs of the terms in the Navier-Stokes equation, the variance and flatness values are presented in Tables III and IV, respectively.

As expected, the pdf of the velocity field is quasi-Gaussian, as shown in Fig. 7 (top, left). By definition, the variance of the velocity field is twice the turbulent kinetic energy. Hence, the variance of the velocity pdfs changes according to the evolution of the turbulent kinetic energy and the largest value is found for the case with the strongest growth for $f / S=+0.5$. 

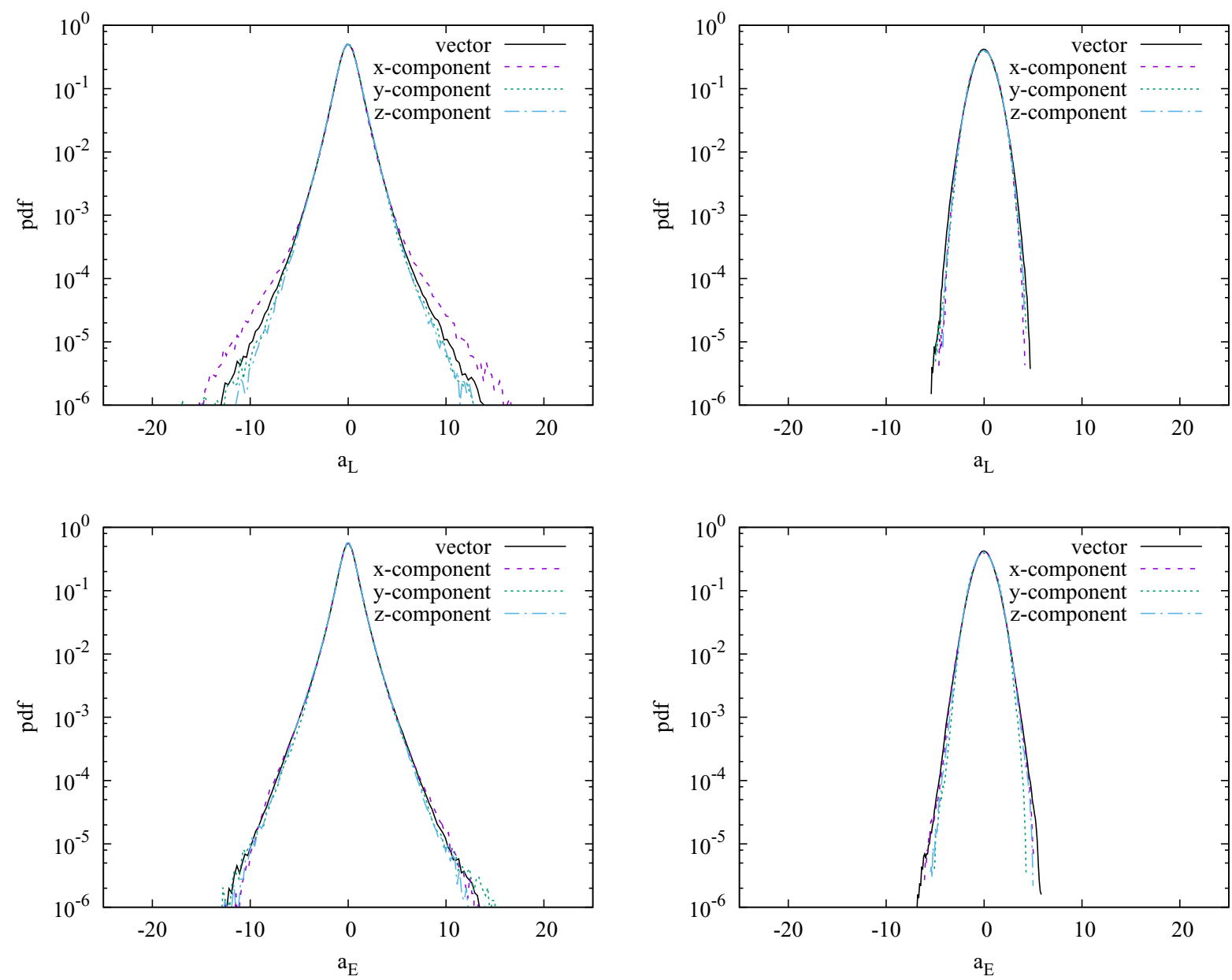

FIG. 6. Normalized pdfs of Lagrangian (top) and Eulerian (bottom) accelerations for $f / S=+0.5$ (left) and $f / S=+5$ (right).

Since the shape of the velocity pdfs remains quasi-Gaussian, the flatness values remain close to three.

Figure 7 (top, right) shows that the pdfs of the viscous term have a stretched exponential behavior. The variance of the viscous term remains small compared to those of other terms in the Navier-Stokes equations and the flatness assumes values between five and seven.

The pdfs of the shear term in Fig. 7 (center, left) and of the rotation term in Fig. 7 (center, right) are quasi-Gaussian, because the corresponding terms in the Navier-Stokes equation

TABLE II. Ratio of the component variance to the total variance of the vector field to quantify the anisotropy of Lagrangian and Eulerian acceleration components at nondimensional time $\mathrm{St}=5$. A value of $1 / 3$ corresponds to isotropy.

\begin{tabular}{lrrrrr}
\hline \hline$f / S$ & -5 & -0.5 & 0 & +0.5 & +5 \\
\hline$a_{L x}^{2} / a_{L}^{2}$ & 0.434 & 0.335 & 0.317 & 0.263 & 0.426 \\
$a_{L y}^{2} / a_{L}^{2}$ & 0.345 & 0.297 & 0.300 & 0.359 & 0.395 \\
$a_{L z}^{2} / a_{L}^{2}$ & 0.221 & 0.368 & 0.383 & 0.378 & 0.180 \\
$a_{E x}^{2} / a_{E}^{2}$ & 0.436 & 0.389 & 0.346 & 0.273 & 0.426 \\
$a_{E y}^{2} / a_{E}^{2}$ & 0.330 & 0.226 & 0.258 & 0.278 & 0.371 \\
$a_{E z}^{2} / a_{E}^{2}$ & 0.235 & 0.385 & 0.397 & 0.449 & 0.203 \\
\hline \hline
\end{tabular}

are proportional to velocity. Note that the shear term is only nonzero for the $x$ component of the Navier-Stokes equation, while the rotation term only contributes to the $x$ and $y$ components. Hence, one or two of the vector components are zero, and those vanishing components have been excluded from the computation of the pdfs and flatness to avoid biasing of the results. The variance, however, is computed from all three components.

The variance of the shear term follows the variance of the velocity, because the shear rate is constant in the simulations. For the cases with growing turbulent kinetic energy with $f / S=0$ and $f / S=+0.5$, the shear term is stronger than the rotation term. For the cases with strong rotation with $f / S= \pm 5$, the importance of the shear term is reduced, while the variance of the rotation term is increased, confirming the dominance of rotation over shear. The flatness of the shear and rotation terms are always close to three as they follow quasi-Gaussian distributions.

The pdfs of the pressure-gradient term in Fig. 7 (bottom, left) and of the nonlinear term in Fig. 7 (bottom, right) exhibit both stretched-exponential behavior. Only the strongly rotating cases with $f / S= \pm 5$ show an approximately Gaussian shape for the pressure-gradient pdfs. Note that the source term of the Poisson equation contains contributions of shear and rotation. Due to the dominance of the rotation term 

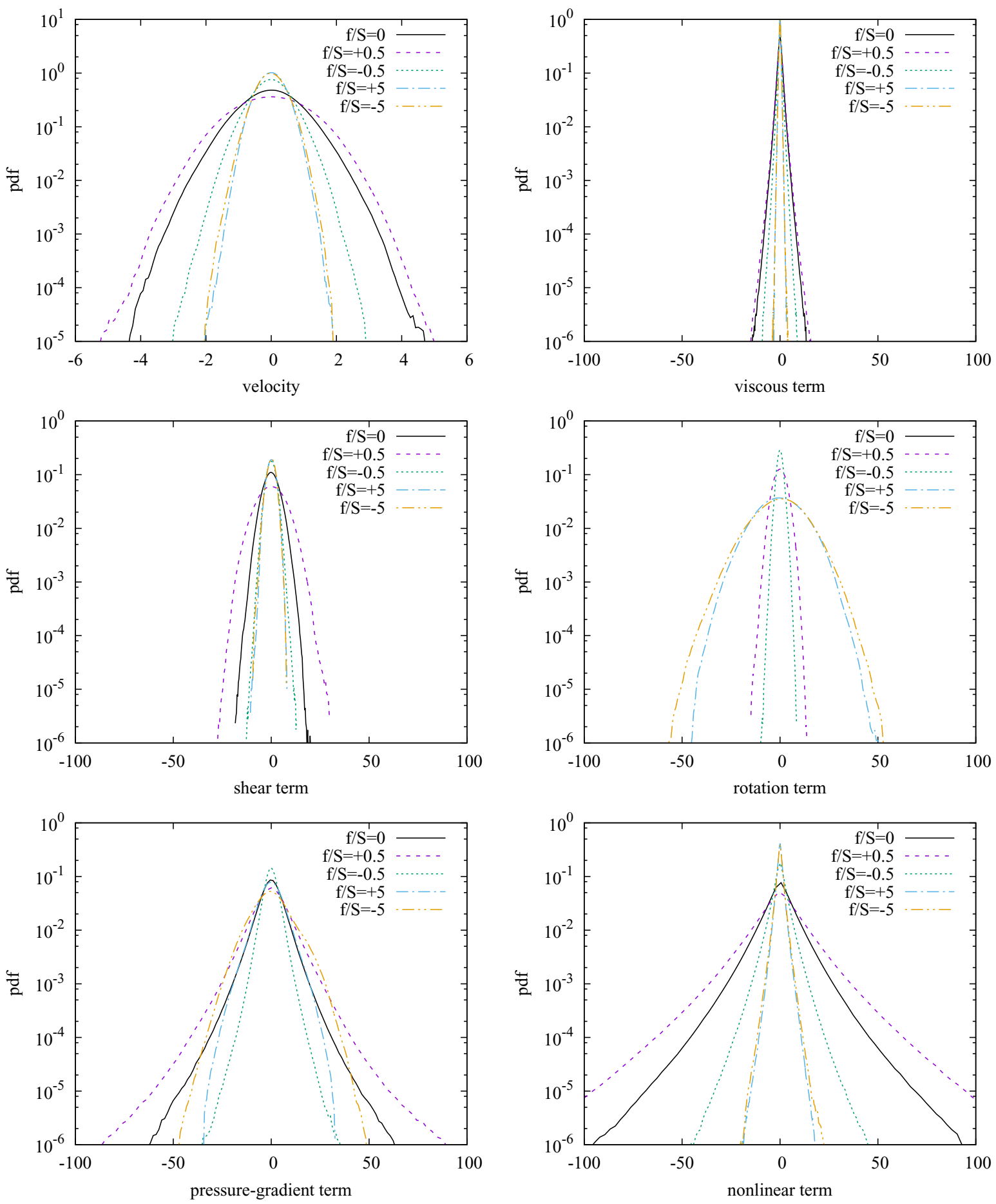

FIG. 7. Pdfs of velocity (top, left), viscous term (top, right), shear term (center, left), rotation term (center, right), pressure-gradient term (bottom, left), and nonlinear term (bottom, right).

for $f / S= \pm 5$, flatness values smaller than six of the pressure gradient are obtained.

For the nonlinear term, we again find that the variance is strongest for the growing case with $f / S=+0.5$ and, as expected, the smallest for $f / S= \pm 5$ because linear effects dominate, as discussed in Ref. [21]. Consequently, for these two cases, the variances of the Lagrangian and Eulerian accelerations are almost identical due to the small variance observed for the nonlinear term.
However, the accelerations are different for the two strongly rotating cases: The parallel configuration with $f / S=-5$ exhibits a much larger variance value as compared to the antiparallel case with $f / S=+5$. This is due to a stronger rotation term for $f / S=-5$, where the system vorticity and shear vorticity are parallel and thus add. Note that the Coriolis force is a fictitious (or pseudo-) force without an effect on the energy, which is reflected in the almost equal variances of the velocity field for the two cases. The large rotation term 
TABLE III. Variance of velocity, viscous term, shear term, rotation term, pressure-gradient term, nonlinear term, Lagrangian acceleration, and Eulerian acceleration, for five values of the rotation to shear ratio $f / S$.

\begin{tabular}{lrrrrr}
\hline \hline & \multicolumn{5}{c}{$f / S$} \\
\cline { 2 - 6 } Term & -5 & -0.5 & 0 & +0.5 & +5 \\
\hline $\boldsymbol{u}$ & 0.17 & 0.32 & 0.77 & 1.19 & 0.15 \\
$\nu \nabla^{2} \boldsymbol{u}$ & 0.16 & 0.66 & 1.46 & 1.73 & 0.15 \\
$S v \boldsymbol{e}_{x}$ & 1.41 & 1.72 & 4.65 & 14.05 & 1.41 \\
$2 \boldsymbol{\Omega} \times \boldsymbol{u}$ & 90.62 & 1.57 & - & 6.46 & 77.30 \\
$\nabla p / \rho_{0}$ & 79.89 & 11.60 & 36.34 & 73.13 & 35.34 \\
$\boldsymbol{u} \cdot \boldsymbol{\nabla} \boldsymbol{u}$ & 3.05 & 14.68 & 78.27 & 174.24 & 2.56 \\
$\boldsymbol{a}_{L}$ & 39.05 & 8.98 & 34.50 & 72.15 & 25.09 \\
$\boldsymbol{a}_{E}$ & 40.84 & 11.43 & 51.58 & 117.28 & 26.49 \\
$\Lambda^{2}$ (DNS estimate) & 92.19 & 3.95 & 6.11 & 22.24 & 78.86 \\
$\Lambda^{2}[$ Eq. (28)] & 108.47 & 8.37 & 8.05 & 12.45 & 64.33 \\
$\Pi_{L}^{2}[$ Eq. (31)] & 54.06 & 3.85 & 3.22 & 1.86 & 32.01 \\
\hline \hline
\end{tabular}

is also responsible for the large pressure-gradient term and the divergence of the Coriolis force is a source term on the right-hand side of the Poisson equation for pressure.

Finally, the Lagrangian and Eulerian accelerations have flatness values close to three due to the dominance of the rotation term for $f / S= \pm 5$. This result differs from the finding
TABLE IV. Flatness of velocity, viscous term, shear term, rotation term, pressure-gradient term, nonlinear term, Lagrangian acceleration, and Eulerian acceleration, for five values of the rotation to shear ratio $f / S$.

\begin{tabular}{lccccc}
\hline \hline & \multicolumn{5}{c}{$f / S$} \\
\cline { 2 - 6 } Term & -5 & -0.5 & 0 & +0.5 & +5 \\
\hline $\boldsymbol{u}$ & 3.14 & 3.39 & 3.36 & 2.93 & 3.02 \\
$v \nabla^{2} \boldsymbol{u}$ & 4.78 & 6.50 & 7.16 & 7.35 & 5.01 \\
$S v \boldsymbol{e}_{x}$ & 3.04 & 3.15 & 3.16 & 2.90 & 2.87 \\
$2 \boldsymbol{\Omega} \times \boldsymbol{u}$ & 3.19 & 3.65 & - & 2.92 & 2.97 \\
$\nabla p / \rho_{0}$ & 3.74 & 6.03 & 7.22 & 7.46 & 4.37 \\
$\boldsymbol{u} \cdot \nabla \boldsymbol{u}$ & 8.65 & 9.61 & 9.71 & 9.36 & 8.27 \\
$\boldsymbol{a}_{L}$ & 3.27 & 6.34 & 7.15 & 7.37 & 3.24 \\
$\boldsymbol{a}_{E}$ & 3.36 & 6.38 & 8.86 & 8.55 & 3.41 \\
\hline \hline
\end{tabular}

of a value of six characteristic for a Laplace distribution. Using a Gaussian velocity field as a model for isotropic turbulence, Holzer and Siggia [11] have shown that the nonlinear quadratic term on the right-hand side of the Poisson equation for pressure results in exponential tails of the pressure and its gradient.

A comparison with the accelerations in Fig. 2 illustrates that the Lagrangian acceleration is mostly determined by the contribution of the pressure-gradient term, while the Eulerian
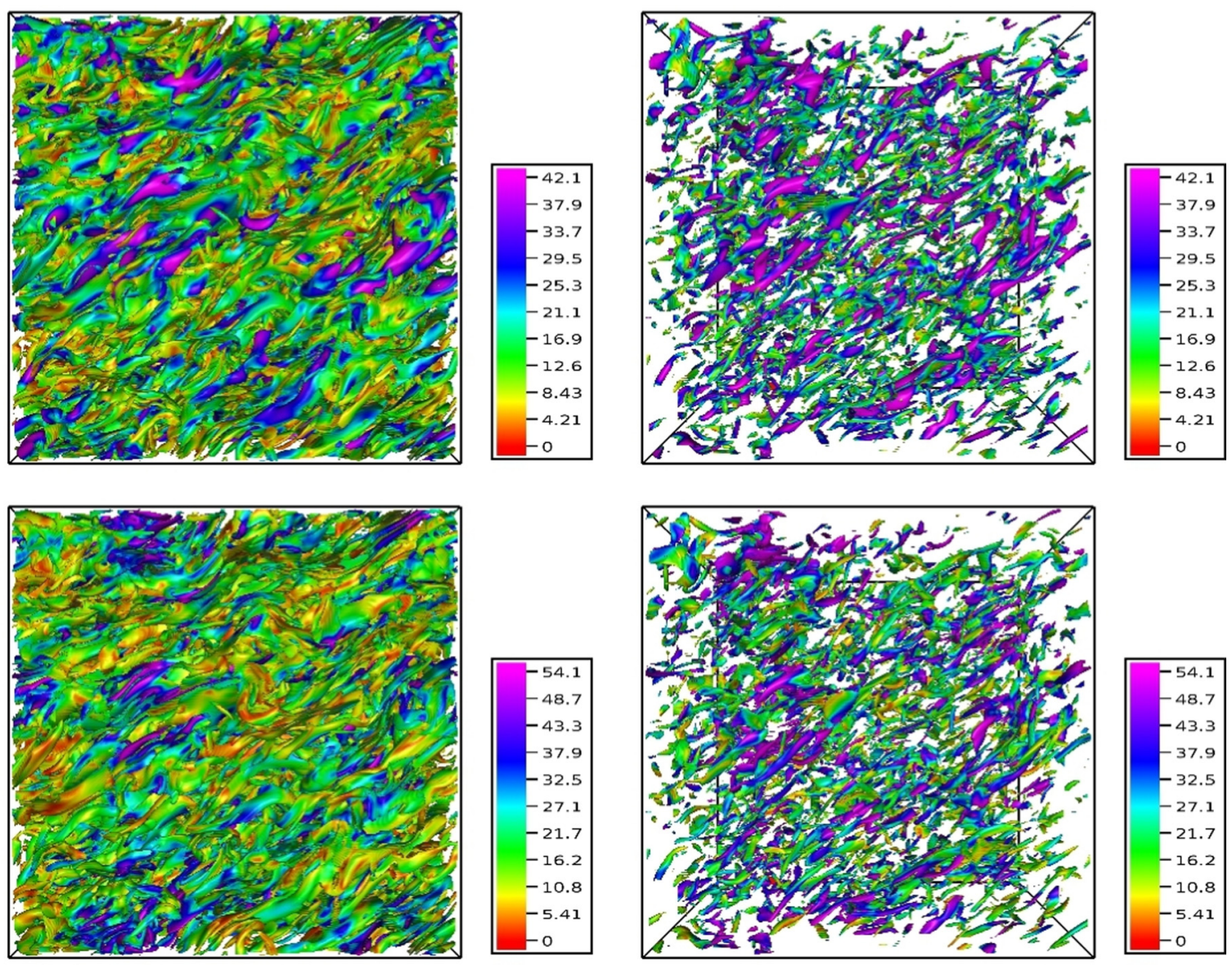

FIG. 8. Isosurfaces of vorticity magnitude at $|\omega|=2 \sigma$ (left) and $|\omega|=4 \sigma$ (right) colored with the magnitude of Lagrangian acceleration (top) and Eulerian acceleration (bottom) for $f / S=+0.5$ at $S t=5$. The view is onto the plane of shear with the $x$ direction to the right and the $y$ direction up. 

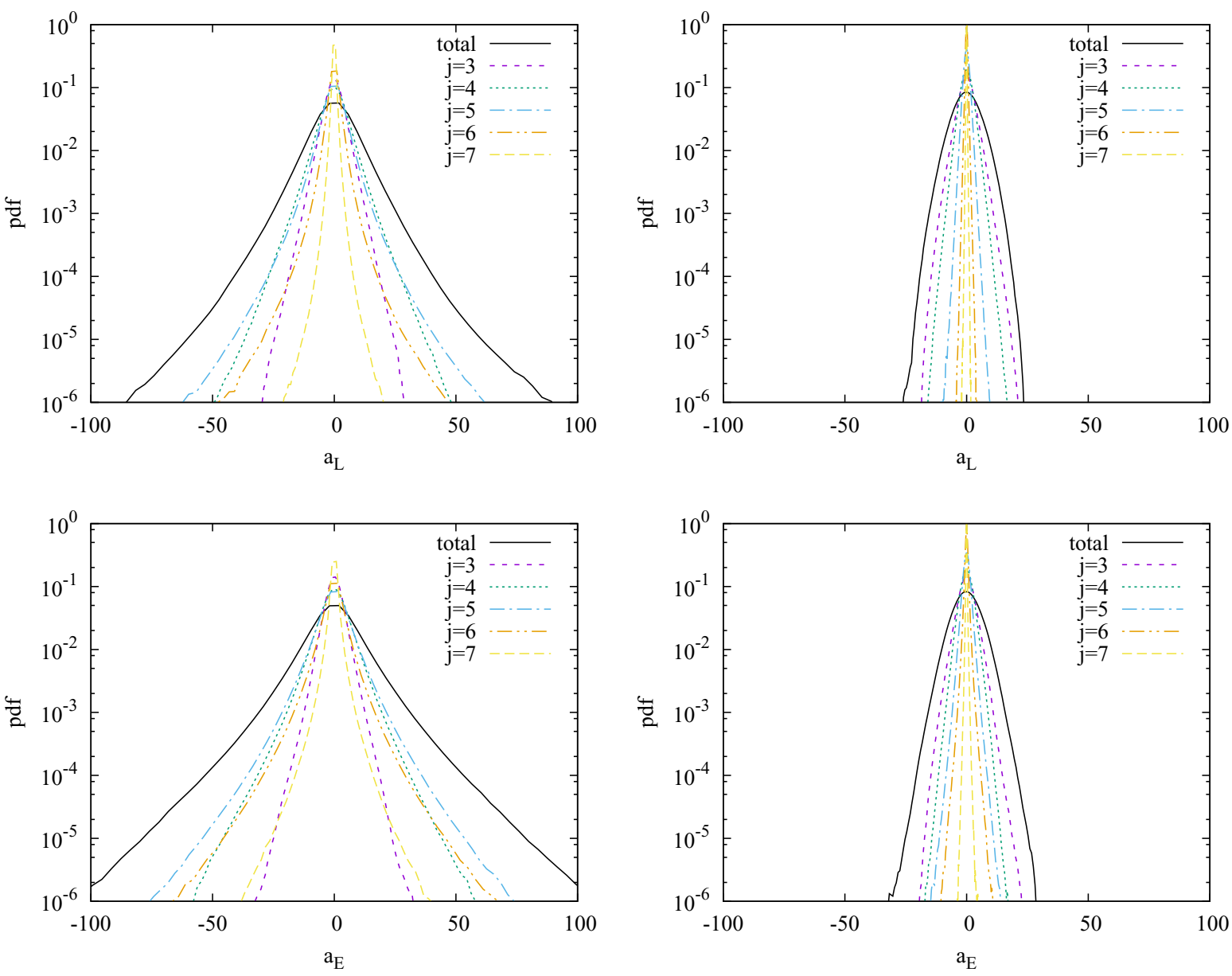

FIG. 9. Scale-dependent pdfs ( $j=3$ corresponds to large scale and $j=7$ to the smallest scale) of Lagrangian (top) and Eulerian (bottom) acceleration for $f / S=+0.5$ (left) and $f / S=+5$ (right) together with the pdf of the total fields.

acceleration is mostly determined by the nonlinear term (except for the strongly rotating cases with $f / S= \pm 5$ ).

In the simulations the shear-number varies from two to about six. For these moderate values, we cannot expect the inequalities (35) and (36) to hold, so that the variance of the acceleration is not directly dominated by the influence of shear. Indirectly, however, this is the case, since the shear acts as an energy input to the system. Indeed, we see in Table III that the shear contribution to the acceleration is never the dominant term for all simulations. The rotation rate is considerably stronger for the cases $f / S= \pm 5$, and its effect is therefore dominant in at least the Lagrangian acceleration and perhaps also in the Eulerian acceleration.

The shape of the pdfs of the Eulerian and Lagrangian accelerations is consistent with the scaling arguments. For the cases in which the dynamics are dominated by linear effects, for the cases $f / S= \pm 5$, the pdfs become Gaussian, reflecting the Gaussian character of the velocity. Also, the predicted variance of the linear terms $\Lambda^{2}$ follows the trend of the DNS estimate, which is computed as an upper bound as the sum of the variances of the viscous, shear, and rotation terms (Table III).

The scaling behavior of the nonlinear term, compared to the nonlinear pressure-gradient term is not verifiable, since the pressure in the simulations contains both linear and nonlinear contributions. However, for the case with $f / S=0$, where linear effects are weak, it is clearly observed that the nonlinear term is stronger than the pressure term. Hence, the predicted variance of the pressure-gradient term based on linear contributions only, $\Pi_{L}^{2}$, compares well with the actual variance of the pressure-gradient term for the cases with $f / S= \pm 5$ (Table III). This comparison fails for the remaining cases in which nonlinear effects dominate the dynamics.

We also refer to Appendix A, where the acceleration pdfs and the contributions of the different terms in the NavierStokes equation have been analyzed for isotropic turbulence.

\section{Scale-dependent analysis}

In order to motivate a scale analysis of the acceleration statistics, Fig. 8 shows isovorticity surfaces at twice (left) and four times (right) the vorticity variance, colored with Lagrangian (top) and Eulerian (bottom) accelerations for the most strongly growing case with $f / S=+0.5$ at nondimensional time $\mathrm{St}=5$. We observe inclined vortical structures. A comprehensive discussion of the inclination angle can be found in Jacobitz et al. [18]. Doubling the isovalues and hence considering stronger and larger vortical structures, we see larger values of both Lagrangian and Eulerian accelerations. Furthermore, locations with strong Lagrangian and Eulerian 

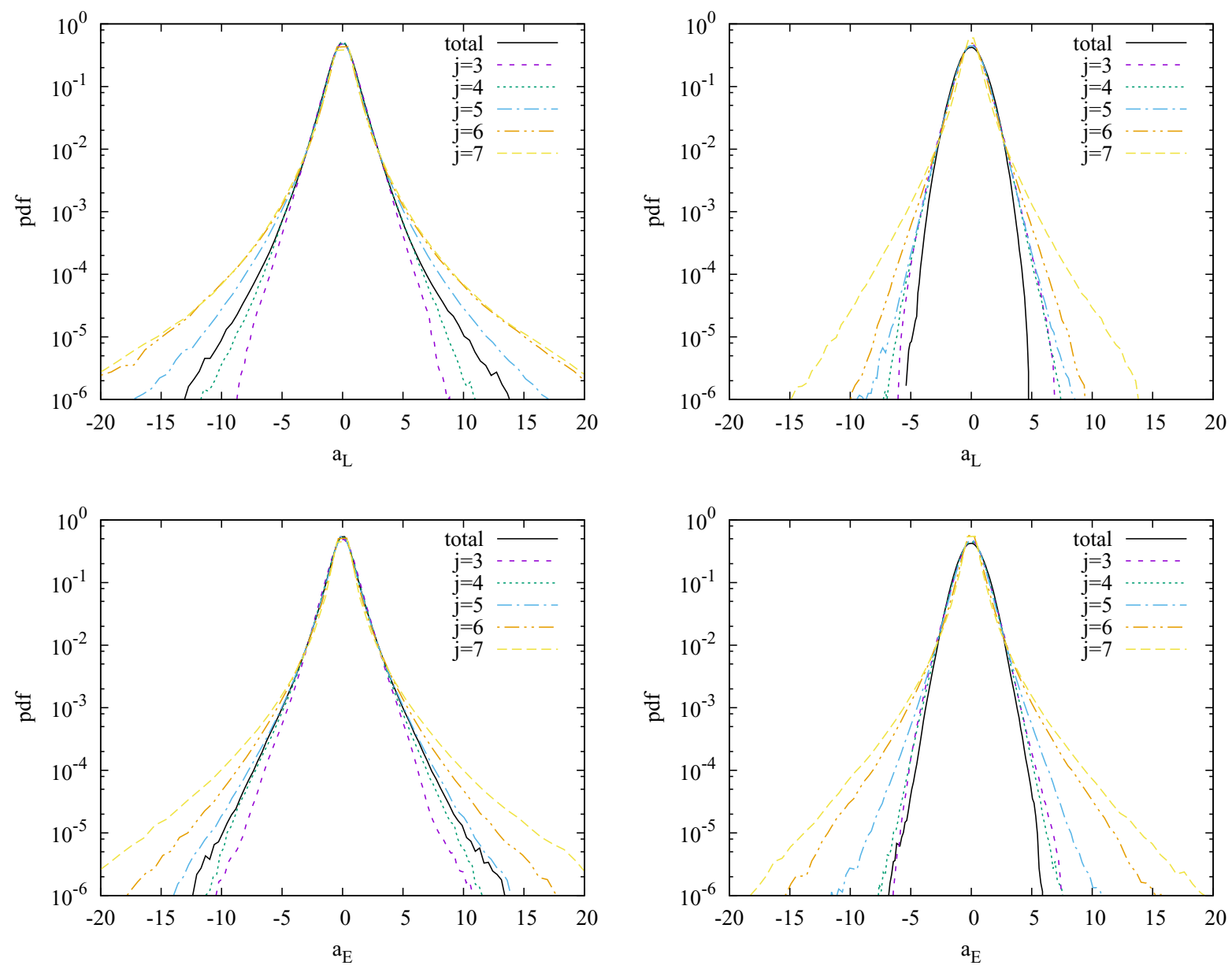

FIG. 10. Normalized scale-dependent pdfs of Lagrangian (top) and Eulerian (bottom) acceleration for $f / S=+0.5$ (left) and $f / S=+5$ (right).

accelerations often coincide, in agreement with the joint pdfs shown in Fig. 5 and the Pearson product-moment correlation coefficient given in Table I.

The scale-dependent Lagrangian or Eulerian accelerations are obtained by decomposing the vector $\boldsymbol{a}=\left(a^{1}, a^{2}, a^{3}\right)$ with $\boldsymbol{a}=\boldsymbol{a}_{L}$ or $\boldsymbol{a}_{E}$, respectively, given at resolution $N=2^{3 J}$ with $J=8$, into an orthogonal wavelet series using Coiflet 12

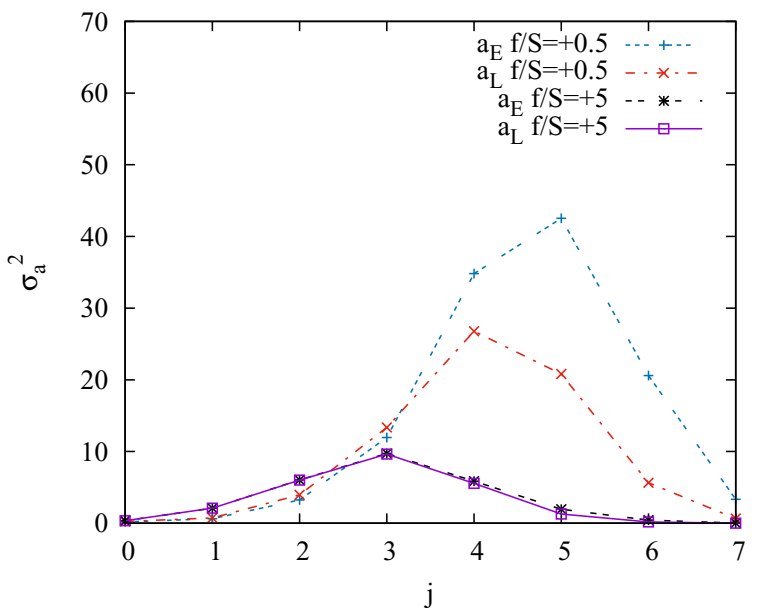

wavelets

$$
\boldsymbol{a}(\boldsymbol{x})=\sum_{\lambda} \widetilde{\boldsymbol{a}}_{\lambda} \psi_{\lambda}(\boldsymbol{x}),
$$

where the multi-index $\lambda=(j, \mathbf{i}, \mu)$ denotes scale index $j$ (with $0 \leqslant j \leqslant J-1$ ), spatial position $\mathbf{i}$ (with $2^{3 j}$ values for each $j$ and $\mu$ ), and seven spatial directions $\mu=1, \ldots, 7$ of

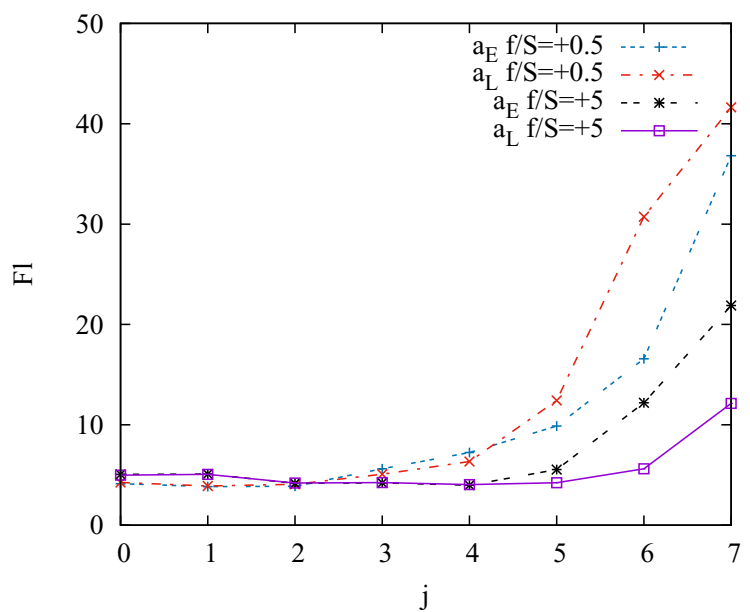

FIG. 11. Variance (left) and flatness (right) of Lagrangian and Eulerian acceleration as a function of scale index $j$. 
TABLE V. Bradshaw number $B=f / S(f / s-1)$, large-scale Rossby number $\mathrm{Ro}_{L}=\epsilon /(2 f K)$, and small-scale Rossby number $\mathrm{Ro}_{s}=\omega / f$ at nondimensional time $\mathrm{St}=5$.

\begin{tabular}{lccccc}
\hline \hline$f / S$ & -5 & -0.5 & 0 & +0.5 & +5 \\
\hline$B$ & 30 & 0.75 & 0 & -0.25 & 20 \\
$\operatorname{Ro}_{L}$ & -0.0171 & -0.244 & $\infty$ & 0.166 & 0.0169 \\
$\operatorname{Ro}_{s}$ & -0.285 & -4.645 & $\infty$ & 7.440 & 0.269 \\
\hline \hline
\end{tabular}

each wavelet $\psi_{\lambda}$ [15]. Orthogonality implies that the wavelet coefficients are given by $\tilde{\boldsymbol{a}}_{\lambda}=\left\langle\boldsymbol{a}, \psi_{\lambda}\right\rangle$, where $\langle\cdot, \cdot\rangle$ denotes the $L^{2}$-inner product. The coefficients measure fluctuations of $\boldsymbol{a}$ at scale $2^{-j}$ and around position $\mathbf{i} / 2^{j}$ for each of the 7 possible directions. Fixing $j$ and summing only over $\mathbf{i}$ and $\mu$, the contribution of $\boldsymbol{a}$ at scale $j$ is obtained, and by construction we have $\boldsymbol{a}=\sum_{j} \boldsymbol{a}_{j}$. Note that the scalewise contributions $\boldsymbol{a}_{j}$ are orthogonal to each other, and hence the total variance of $\boldsymbol{a}$ is obtained by summing the variances of $\boldsymbol{a}_{j}$.

For the wavelet-based scale-dependent analysis, two typical cases, one with moderate rotation to shear ratio $f / S=+0.5$ and another with strong rotation to shear ratio $f / S=+5$, are considered. Figure 9 shows the scale-dependent pdfs of Lagrangian acceleration (top) and Eulerian acceleration (bottom) for $f / S=+0.5$ (left) and $f / S=+5$ (right). For the growing case with $f / S=+0.5$, a stretched-exponential-like behavior is observed for the Lagrangian acceleration at most scales (excluding the largest scales), while the decaying case with $f / S=+5$ shows Gaussian-like behavior at all scales. The corresponding Eulerian accelerations for cases with $f / S=$ +0.5 and $f / S=+5$ show similar features. However, the tendency for larger extreme values of the Eulerian acceleration observed for the total pdf persists at all scales.

Figure 10 shows the normalized scale-dependent pdfs of both Lagrangian (top) and Eulerian (bottom) accelerations for $f / S=+0.5$ (left) and $f / S=+5$ (right). The shapes of both accelerations vary with scale. With decreasing scale, i.e., increasing scale index $j$, the tails become heavier. Again, heavier tails are present at all scales for the case $f / S=+0.5$, which reflect the stronger intermittency of the flow.

The scale-dependent flatness of both the Lagrangian and Eulerian accelerations for the two $f / S$ values (see Fig. 11) shows a strong increase for decreasing scale (increasing $j$ ), which quantifies that small-scale contributions are more intermittent than large-scale contributions. For $f / S=+0.5$, the flatness of the Lagrangian acceleration is larger than the flatness of the Eulerian acceleration (for $j<4$ ), similar to observations for isotropic turbulence in Yoshimatsu et al. [12]. In the case $f / S=+5$, the values of the Eulerian acceleration are larger than the values of the Lagrangian acceleration (for $j>4$ ), which must be due to the fact that the effect of the nonlinear term becomes weaker for increasing rotation.

The Bradshaw number together with large and small scale Rossby numbers at the nondimensional time $\mathrm{St}=5$ are assembled in Table V. The Bradshaw number is often used as an alternative to the rotation to shear ratio $f / S$ in the description of rotating turbulence. However, it assumes the same value for $f / S=0$ and $f / S=1$, while the flows evolve quite differently for the two cases [21]. The large-scale Rossby number $\mathrm{Ro}_{L}$ remains small for all cases considered here, except the case without rotation $f / S=0$. The smallscale Rossby number $\mathrm{Ro}_{s}$, however, assumes somewhat larger values for the cases with moderate rotation $f / S= \pm 0.5$.

\section{Passive scalar structure and dynamics}

In order to gain more insight into the structure and dynamics of turbulence with shear and rotation, the evolution of a passive scalar with a vertical mean gradient is considered in this section. In the context of homogeneous turbulent shear flows with rotation, Brethouwer [20] studied the dynamics of passive scalars. Here, we focus on the Lagrangian and Eulerian time rate of change terms in the advection-diffusion equation for a scalar in analogy to the Lagrangian and Eulerian acceleration terms in the Navier-Stokes equation.

Figure 12 shows a volume visualization of the fluctuating scalar for two cases with rotation to shear ratios $f / S=+0.5$ (left) and $f / S=+5$ (right) at nondimensional time $S t=5$. In

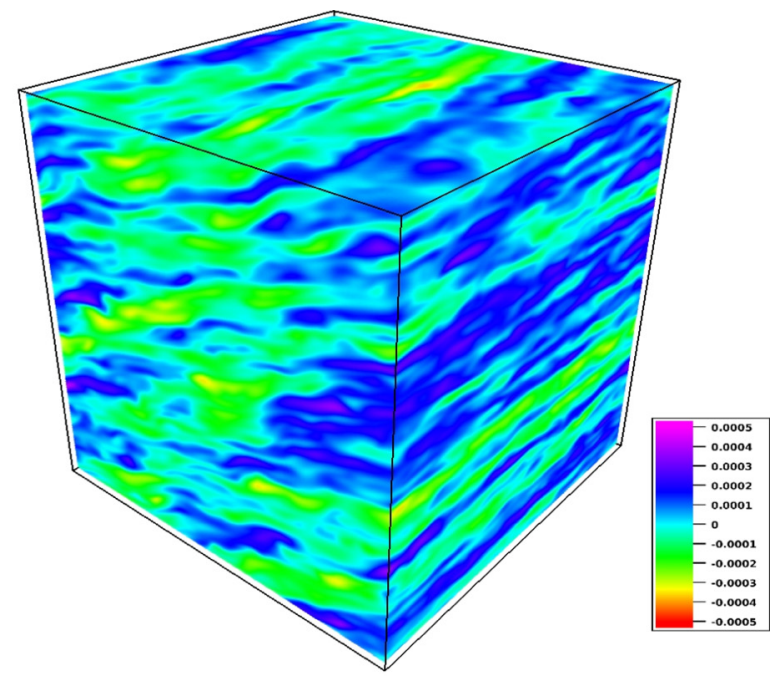

FIG. 12. Volume visualization of the fluctuating scalar component for $f / S=+0.5$ (left) and $f / S=+5$ (right). 

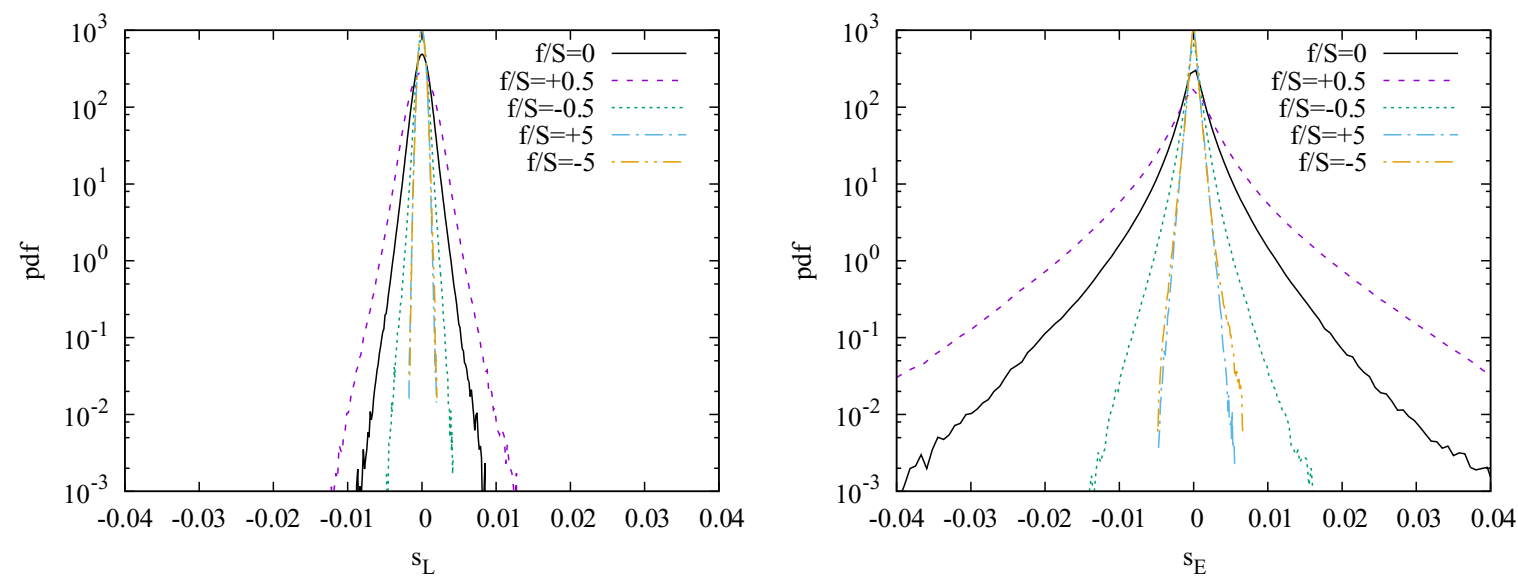

FIG. 13. Pdfs of Lagrangian (left) and Eulerian (right) time rate of change.

both cases, the view onto the plane of shear (view from the left onto the $x y$ plane) shows structures inclined to the downstream $x$ direction in the vertical $y$ direction due to the action of shear. This effect is similar to previous results for inclined vortex structures discussed previously [18]. For strong rotation with $f / S=+5$, the fluctuating scalar exhibits inclined sheetlike structures, while moderate rotation with $f / S=+0.5$ results in a less organized distribution of the scalar fluctuation in the spanwise $z$ direction. The sheetlike structure in the presence of strong rotation implies smaller scalar fluctuations within the plane defined by the sheetlike structures.

Figure 13 shows the pdfs of the Lagrangian time rate of change (left) and the Eulerian time rate of change (right). Again, a strong and similar dependence on the rotation to shear ratio $f / S$ is obtained for both quantities. Figure 14 presents the Lagrangian and Eulerian time rate of change pdfs normalized with their corresponding standard deviation. Two families are obtained for the Lagrangian time rate of change pdf (left): Gaussian-like behavior for strong rotation with $f / S= \pm 5$, and stretched-exponential behavior for the remaining cases. The Eulerian time rate of change pdf (right) generally shows a stretched-exponential-like behavior with a trend towards exponential-like shape for the strongly rotating cases. Hence, the normalized Lagrangian time rate of change pdfs distinguish ranges for linear and advection-dominated scalar dynamics, but no such information can easily be obtained from the Eulerian time rate of change pdfs.

The variances of the Lagrangian and Eulerian time rates of change differ by about a factor of two for the strongly rotating cases with $f / S= \pm 5$ to an order of magnitude for the case with $f / S=+0.5$ (Table VII). In agreement with the findings for the accelerations, the extreme values of the Eulerian time rate of change are above those of the Lagrangian time rate of change. The flatness of the Eulerian time rate of change varies from the Gaussian value of 3 for $f / S= \pm 5$ to a maximum of about 4.5 for $f / S=0$ (Table VIII). The flatness of the Eulerian time rate of change assumes significantly larger values ranging from about 6 to almost 16, indicating a higher level of intermittency in the Eulerian time rate of change as compared to its Lagrangian counterpart.

To check the correlation between Lagrangian and Eulerian time rates of change, we show in Fig. 15 the joint pdfs for a growing case with $f / S=+0.5$ and a decaying case with $f / S=+5$. Again, the strongly rotating case with $f / S=+5$ indicates correlation of the time rates of change, but the correlation is not as pronounced as that observed for the corresponding accelerations. These observations are further quantified with the Pearson product-moment
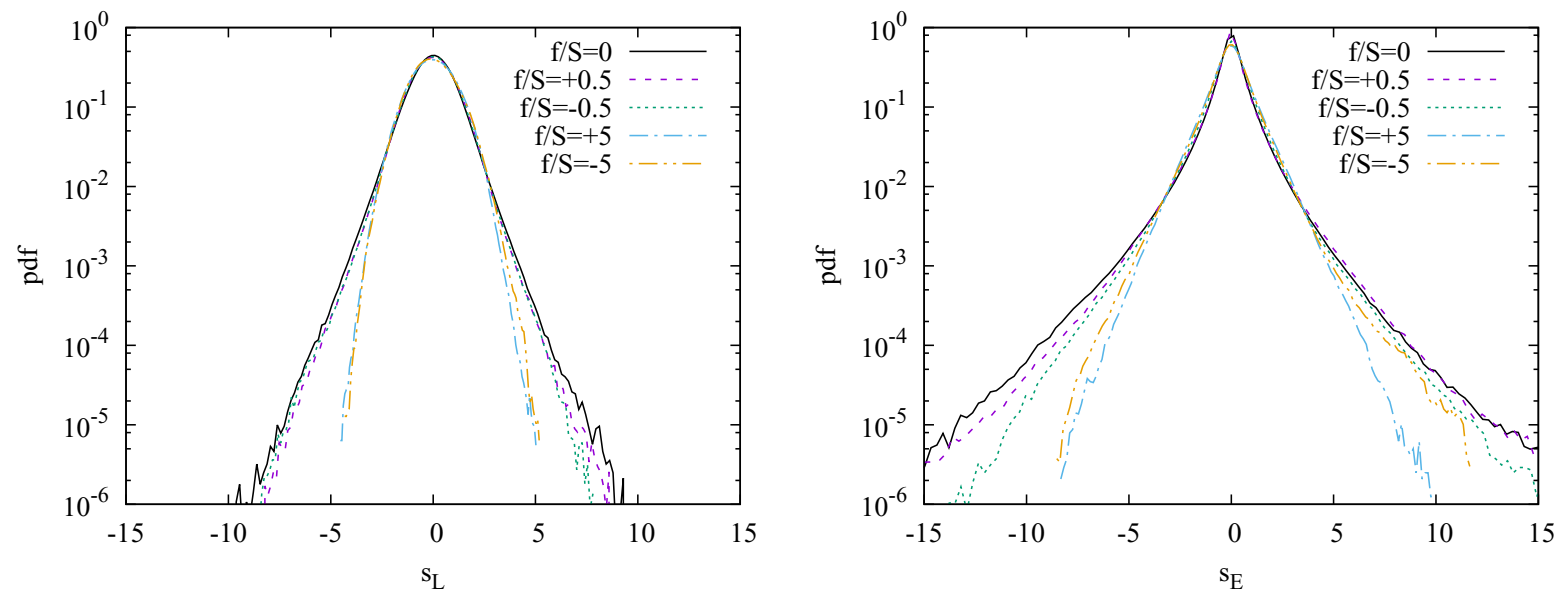

FIG. 14. Normalized pdfs of Lagrangian (left) and Eulerian (right) time rate of change. 

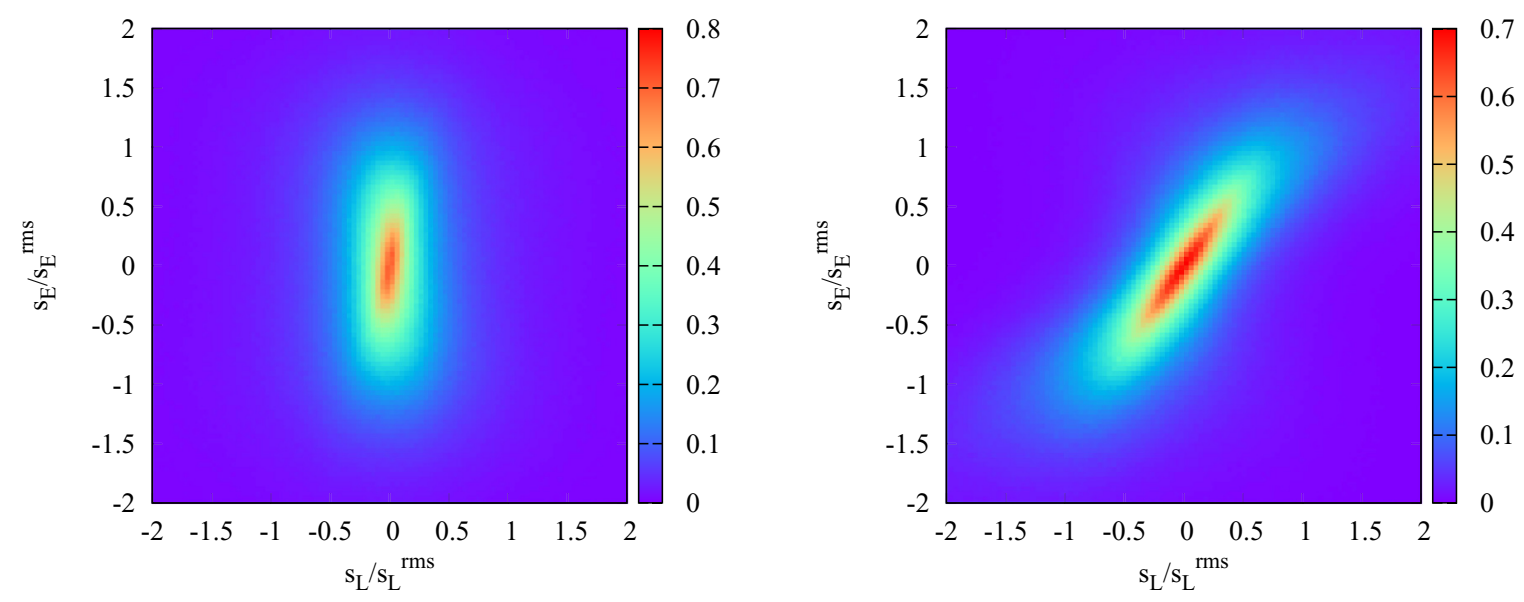

FIG. 15. Joint pdfs of the time rate of change of fluctuating density for $f / S=+0.5$ (left) and $f / S=+5$ (right).

correlation coefficient $r$, given in Table VI. The correlation coefficient for the Lagrangian and Eulerian time rates of change shows a similar trend as that observed for the accelerations. Again, the correlation coefficient remains small for the growing cases with $f / S=0$ and $f / S=+0.5$, which indicates a lack of correlation between the time rates of change, and a moderate value is obtained for the case with $f / S=$ -0.5 . The strongly rotating cases with $f / S= \pm 5$ exhibit the largest correlation coefficients, indicating a correlation of the Lagrangian and Eulerian time rates of change. However, these correlation coefficients for the time rates of change are smaller than those observed for the accelerations. Again, a diminished advection term is the main reason for the increased correlation between Lagrangian and Eulerian time rates of change in the case of strong rotation.

In order to further explain the properties of the Lagrangian and Eulerian time rates of change, pdfs of the fluctuating scalar and all terms in the advection-diffusion equation for its evolution are presented in Fig. 16. The variance and flatness values of the pdfs are provided in Tables VII and VIII, respectively.

The pdfs of the fluctuating scalar are shown in Fig. 16 (top, left) and they have, as expected, a quasi-Gaussian shape. The variance of the fluctuating scalar roughly follows that of the velocity and the strongest scalar fluctuations are observed for the strongly growing case with $f / S=+0.5$. The lowest scalar fluctuations are observed for the strongly rotating cases with $f / S= \pm 5$. Confirming their quasi-Gaussian shapes, the flatness of the pdfs is close to three for all cases.

As there is no analogon to the pressure-gradient term present in the advection-diffusion equation for the fluctuating scalar, the Lagrangian time rate of change is completely determined by linear effects due to the scalar viscous and

TABLE VI. Pearson product-moment correlation coefficient $r$ for the Lagrangian and Eulerian time rates of change at nondimensional time $\mathrm{St}=5$.

\begin{tabular}{lccccc}
\hline \hline$f / S$ & -5 & -0.5 & 0 & +0.5 & +5 \\
\hline$r$ & 0.6388 & 0.2505 & 0.0939 & 0.0759 & 0.6441 \\
\hline \hline
\end{tabular}

production terms. The pdfs of the scalar viscous term generally have a stretched-exponential-like shape, but they tend to a more Gaussian shape for the strongly rotating cases with $f / S= \pm 5$, as shown in Fig. 16 (top, right). The pdfs of the scalar production term, however, always have a quasi-Gaussian shape as shown in Fig. 16 (bottom, left). The variances of the scalar production term are larger than those of the scalar viscous term for strong rotation with $f / S= \pm 5$, but they are fairly balanced for the remaining cases. The flatness of the scalar production term remains always close to 3 , but it varies from about 4 at $f / S= \pm 5$ to a maximum of about 8.5 at $f / S=+0.5$ for the scalar viscous term.

The pdfs of the scalar advection term are presented in Fig. 16 (bottom, right) and they are characterized by stretchedexponential shapes. The flatness of the scalar advection term is comparable to the flatness of the linear terms for the strongly rotating cases with $f / S= \pm 5$ only. For the other cases, however, the scalar advection term is an order of magnitude larger than the linear terms. Hence, it dominates the Eulerian time rate of change.

\section{CONCLUSIONS}

In this study, the Lagrangian and Eulerian acceleration statistics of sheared and rotating homogeneous turbulence are evaluated from direct numerical simulations for a variety of rotation to shear ratios $f / S$. The pdfs of both Lagrangian and Eulerian accelerations exhibit a pronounced dependence on $f / S$ and the extreme values of the Eulerian acceleration are observed to be above those of the Lagrangian acceleration. The derived estimates of the variance of the linear term and its contribution to the pressure-gradient agreed reasonably well with the DNS data although they are based on the assumption of isotropy. Using the Pearson product-moment correlation coefficient, the accelerations were found to be strongly correlated in the cases with strong rotation, due to a reduced nonlinear term. The anisotropy of rotating and sheared turbulence is also observed in the components of the Lagrangian and Eulerian accelerations. In the case of strong rotation, the variances of the accelerations are reduced in 

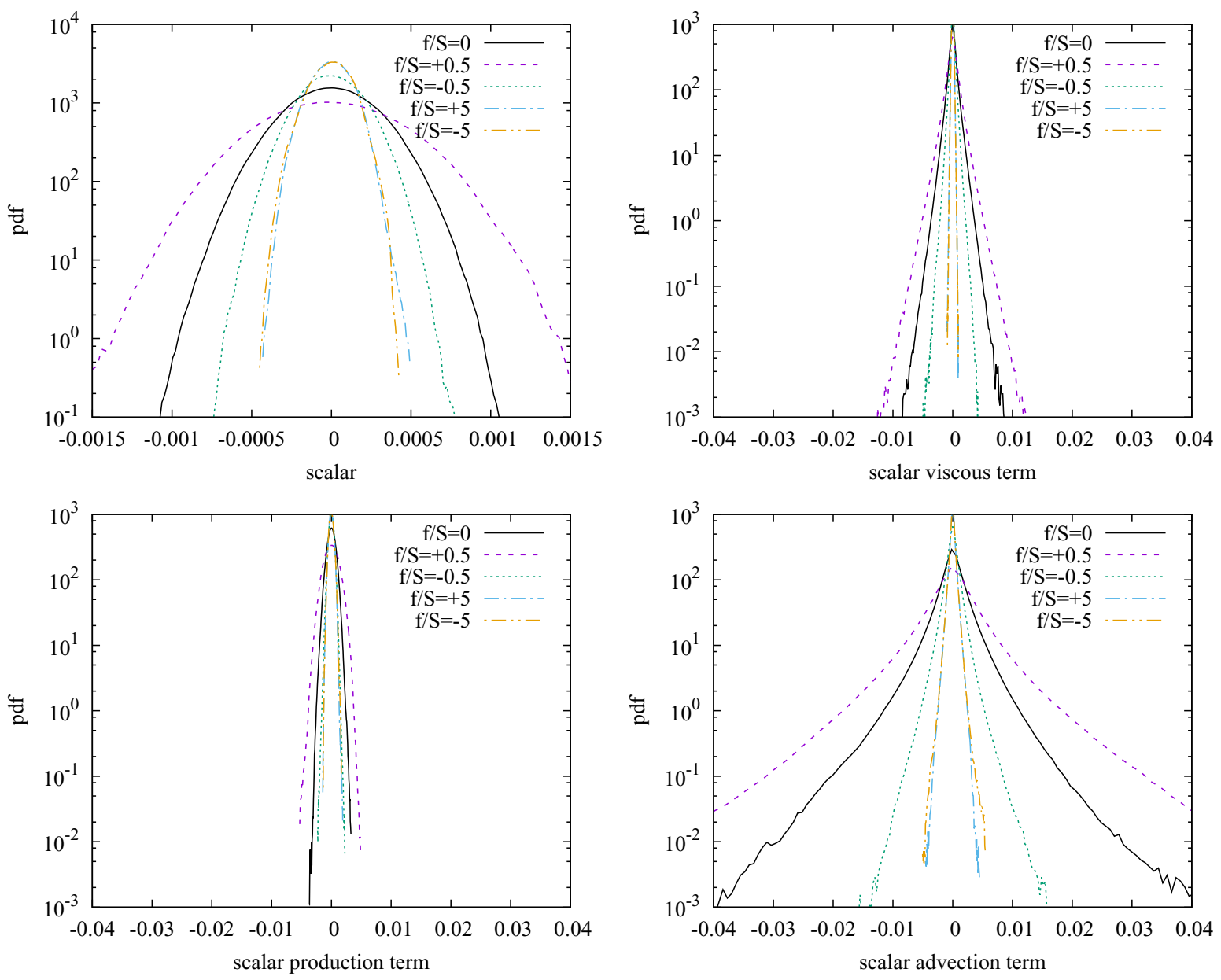

FIG. 16. Pdfs of the fluctuating scalar (top, left), scalar viscous term (top, right), scalar production term (bottom, left), and scalar advection term (bottom, right) as a function of $f / S$.

the direction of the rotation axis. However, the pdfs of the acceleration components exhibit similar shapes.

For both accelerations, the flatness yields values close to three for strong rotation, which indicates a Gaussian-like behavior. For moderate and vanishing rotations, however, the flatness of the Eulerian acceleration is larger than that of the Lagrangian acceleration at the Reynolds number of this study, contrary to previous and present results obtained for isotropic turbulence. A wavelet-based scale-dependent analysis shows that the flatness of both Lagrangian and

TABLE VII. Variance of scalar, scalar viscous term, scalar production term, scalar advection term, Lagrangian time rate of change, and Eulerian time rate of change, for five values of the rotation to shear ratio $f / S$. All values are multiplied with $10^{6}$.

\begin{tabular}{lcccrc}
\hline \hline & \multicolumn{5}{c}{$f / S$} \\
\cline { 2 - 6 } Term & -5 & -0.5 & 0 & +0.5 & +5 \\
\hline$c$ & 0.014 & 0.031 & 0.065 & 0.148 & 0.013 \\
$\alpha \nabla^{2} c$ & 0.019 & 0.141 & 0.454 & 1.036 & 0.020 \\
$S_{c} v$ & 0.135 & 0.165 & 0.445 & 1.343 & 0.135 \\
$\boldsymbol{u} \cdot \nabla c$ & 0.194 & 1.092 & 7.177 & 25.864 & 0.189 \\
$s_{L}$ & 0.151 & 0.289 & 0.836 & 2.200 & 0.152 \\
$s_{E}$ & 0.327 & 1.084 & 6.789 & 24.785 & 0.321 \\
\hline \hline
\end{tabular}

Eulerian accelerations increases as scale decreases and hence their intermittency increases. For strong rotation, as reflected in the scale-dependent flatness, the Eulerian acceleration is more intermittent than the Lagrangian acceleration, while the opposite result is obtained for moderate rotation. These observations suggest that linear theory will likely predict the acceleration statistics correctly in the case of strong rotation, extending the work by Salhi et al. [21].

TABLE VIII. Flatness of scalar, scalar viscous term, scalar production term, scalar advection term, Lagrangian time rate of change, and Eulerian time rate of change, for five values of the rotation to shear ratio $f / S$.

\begin{tabular}{lcrrrr}
\hline \hline & \multicolumn{5}{c}{$f / S$} \\
\cline { 2 - 6 } Term & -5 & -0.5 & 0 & +0.5 & +5 \\
\hline$c$ & 2.89 & 2.92 & 2.95 & 2.91 & 2.81 \\
$\alpha \nabla^{2} c$ & 3.82 & 7.41 & 8.40 & 8.46 & 3.85 \\
$S_{c} v$ & 3.05 & 3.15 & 3.16 & 2.90 & 2.87 \\
$\boldsymbol{u} \cdot \nabla c$ & 9.35 & 11.24 & 15.75 & 14.81 & 7.59 \\
$s_{L}$ & 3.06 & 4.00 & 4.49 & 3.95 & 2.93 \\
$s_{E}$ & 8.16 & 11.79 & 17.72 & 16.76 & 6.12 \\
\hline \hline
\end{tabular}


In addition, the Lagrangian and Eulerian time rates of change of a fluctuating passive scalar are considered. Physically, the Lagrangian time rate of change is determined completely by linear effects, which would ease any modeling efforts. The advection term dominates over the viscous and production terms, enhancing the differences between Lagrangian and Eulerian viewpoints, which are present in the scalar evolution due to the absence of a term analog to the pressure-gradient in the Navier-Stokes equation. However, advection results in more extreme Eulerian time rate of change values due to the transport of scalar packets past a stationary Eulerian observer.

Computations at higher resolution, i.e., $512^{3}$, increasing the Reynolds number by about $50 \%$, showed only minor differences for the Lagrangian and Eulerian acceleration pdfs. Analyzing the pdfs at later nondimensional times, i.e., $\mathrm{St}=10$, also showed that our main findings do not change. Hence we anticipate that the obtained results are robust and generally valid for higher Reynolds numbers.

The relation between Eulerian and Lagrangian statistics remains a challenging and unsolved problem in turbulence that is strongly related to the spatial and temporal flow structures. Kamps et al. [9] proposed a formal connection of the pdfs of velocity increments for statistical stationary homogeneous and isotropic flows. Extending their work could be a perspective to bridge both viewpoints for turbulent rotating shear flows.

\section{ACKNOWLEDGMENTS}

F.G.J. acknowledges support from an International Opportunity Grant from the University of San Diego and the hospitality at Aix-Marseille Université. W.B. and K.S. thankfully acknowledge financial support from the Agence Nationale de la Recherche (France), project SiCoMHD, and M.F. and K.S. from Euratom-FR-FCM contract No. n2 TT.FR.1215.

\section{APPENDIX A: ACCELERATION STATISTICS OF ISOTROPIC TURBULENCE}

In order to compare the results for acceleration statistics of sheared and rotating turbulence to those of isotropic

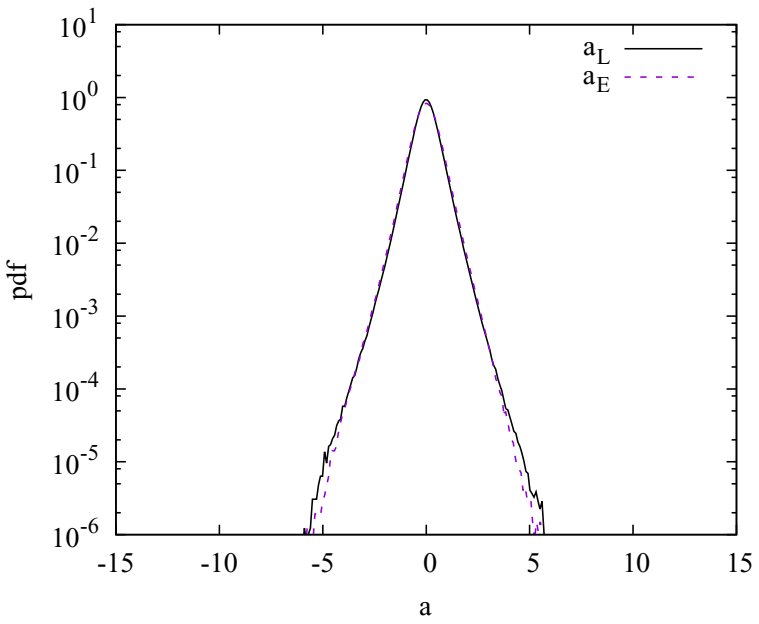

TABLE IX. Variance and flatness of velocity, viscous term, pressure-gradient term, nonlinear term, Lagrangian acceleration, and Eulerian acceleration for isotropic turbulence.

\begin{tabular}{lrr}
\hline \hline Term & Variance & Flatness \\
\hline $\boldsymbol{u}$ & 0.074 & 2.87 \\
$\nu \nabla^{2} u$ & 0.053 & 4.06 \\
$\nabla p / \rho_{0}$ & 0.225 & 6.30 \\
$\boldsymbol{u} \cdot \boldsymbol{\nabla} \boldsymbol{u}$ & 0.540 & 6.08 \\
$\boldsymbol{a}_{L}$ & 0.278 & 5.63 \\
$\boldsymbol{a}_{E}$ & 0.316 & 4.91 \\
$\boldsymbol{a}_{L}$ estimate from Eq. (22) & 0.225 & \\
$\boldsymbol{a}_{E}$ estimate from Eq. (21) & 0.315 & \\
\hline \hline
\end{tabular}

turbulence, an additional simulation without shear nor rotation was performed. This isotropic turbulence simulation was initialized with the same initial condition as the sheared and rotating turbulence cases. The simulation is analyzed at approximately five eddy turn over times. Results for variance and flatness are given in Table IX.

Figure 17 compares the pdfs of Lagrangian and Eulerian accelerations (left) and their normalized counterparts (right). While the shapes of the two pdfs are similar, the pdf of $\boldsymbol{a}_{L}$ shows heavier tails than the pdf of $\boldsymbol{a}_{E}$. In agreement with the estimate (26) and our findings for sheared and rotating turbulence, the variance of $\boldsymbol{a}_{E}$ is larger than the variance of $\boldsymbol{a}_{L}$.

The heavier tails of the pdf of $\boldsymbol{a}_{L}$ indicate that the Lagrangian acceleration is characterized by stronger extreme events as compared to the Eulerian acceleration for isotropic turbulence. These heavier tails result in a larger flatness of $\boldsymbol{a}_{L}$ as compared to the flatness of $\boldsymbol{a}_{E}$. This result differs from our findings for sheared and rotating turbulence at the Reynolds number of this study, but it agrees with previous work: Ishihara et al. [30] found that the flatness of $\boldsymbol{a}_{L}$ is larger than that of $\boldsymbol{a}_{E}$ in forced isotropic turbulence and that this difference is more pronounced at higher Reynolds numbers.

The Pearson product-moment correlation coefficient $r$ based on the three components of Lagrangian and Eulerian accelerations was found to be 0.0911, which indicates a lack of correlation between the accelerations in decaying

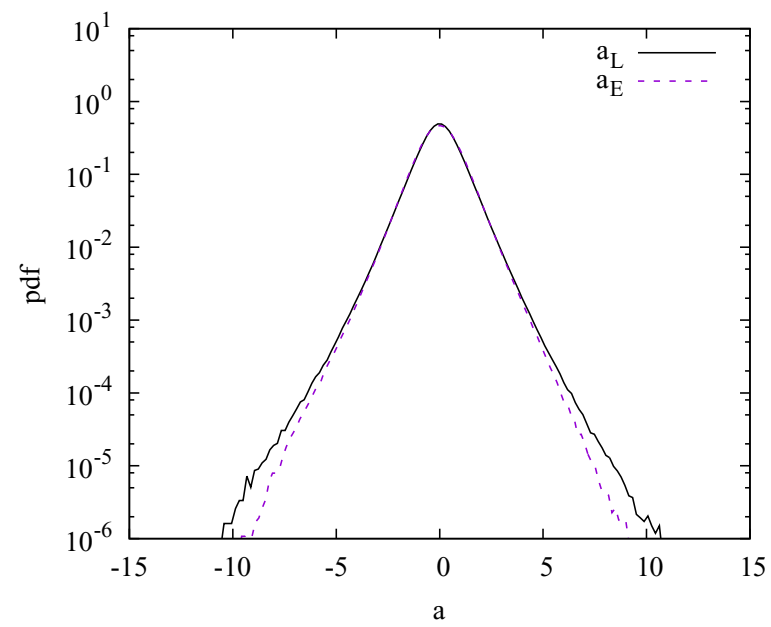

FIG. 17. Pdfs of Lagrangian and Eulerian accelerations of isotropic turbulence (left) and their normalized counterparts (right). 


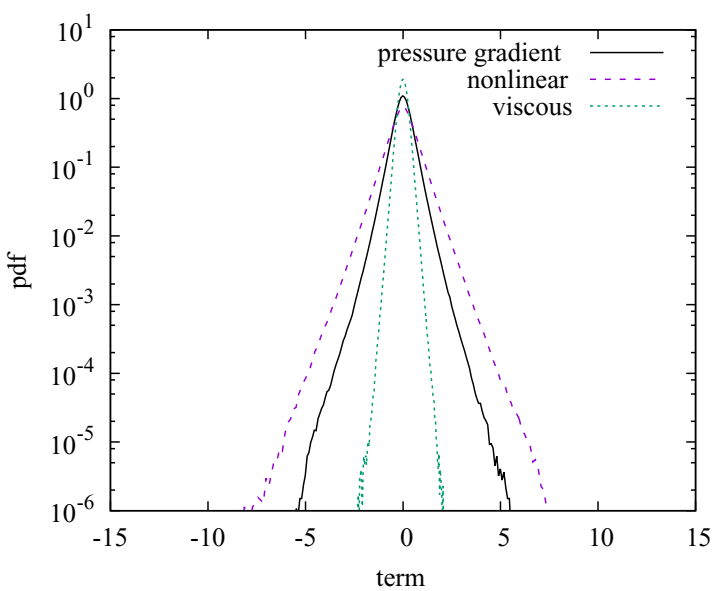

FIG. 18. Pdfs of the viscous term $v \nabla^{2} u$, pressure gradient $\nabla p / \rho_{0}$, and nonlinear term $\boldsymbol{u} \cdot \nabla \boldsymbol{u}$ for isotropic turbulence.

isotropic turbulence. This correlation coefficient is similar to that observed in the growing cases with $f / S=0$ and $f / S=+0.5$. Hence, the correlation between the accelerations observed for strong rotation is indeed due to rotation (and not due to decaying turbulence). The Pearson product-moment correlation coefficient based on the magnitudes of the accelerations assumes a moderate value of 0.2377 for decaying isotropic turbulence.

Figure 18 shows the pdfs of the pressure-gradient, nonlinear term, and viscous term for isotropic turbulence. In agreement with Holzer and Siggia [11], the pdf of the nonlinear term shows exponential tails with a corresponding flatness value of 6.08, which is characteristic for a Laplace distribution. The pdf of the pressure-gradient also yields exponential tails with a flatness value of 6.3. These results can be explained by the fact that the velocity field is almost Gaussian.

Due to the orthogonality of the pressure-gradient to the viscous term, which is the only contributor to the linear term in isotropic turbulence, Eq. (17) can be written as

$$
a_{L}^{2}=\left\langle\|\boldsymbol{\Pi}\|^{2}\right\rangle+\left\langle\|\boldsymbol{\Lambda}\|^{2}\right\rangle
$$

While this equation is exact for isotropic turbulence, the Lagrangian acceleration is dominated by the pressure-gradient term as the variance of the viscous term remains small. Hence, Eq. (22) provides a good estimate for the variance of the Lagrangian acceleration $\boldsymbol{a}_{L}$. The variance of the Eulerian acceleration is also determined well by Eq. (21), and the variance of the Eulerian acceleration $\boldsymbol{a}_{E}$ is given by the difference of the variances of the nonlinear term and the pressure gradient (Table IX).

\section{APPENDIX B: POISSON EQUATION FOR PRESSURE}

To prove expression (14), we Fourier-transform the Poisson equation for pressure (13) to obtain the Fourier coefficients $\widehat{p}$,

$$
\frac{\widehat{p}}{\rho_{0}}=\frac{i k_{j}}{k^{2}}\left(\widehat{N}_{j}+\widehat{\Lambda}_{j}\right)
$$

so that

We obtain that

$$
\widehat{\Pi}_{i}=-\frac{k_{i} k_{j}}{k^{2}}\left(\widehat{N}_{j}+\widehat{\Lambda}_{j}\right) .
$$

$$
\widehat{\Pi}_{i} \widehat{\Pi}_{i}^{*}=\frac{k_{m} k_{n}}{k^{2}}\left(\widehat{N}_{m}+\widehat{\Lambda}_{m}\right)\left(\widehat{N}_{n}+\widehat{\Lambda}_{n}\right)^{*},
$$

where * indicates a complex conjugate. We further have

$$
\widehat{\Pi}_{i}\left(\widehat{N}_{i}+\widehat{\Lambda}_{i}\right)^{*}=-\frac{k_{i} k_{j}}{k^{2}}\left(\widehat{N}_{j}+\widehat{\Lambda}_{j}\right)\left(\widehat{N}_{i}+\widehat{\Lambda}_{i}\right)^{*}=-\widehat{\Pi}_{i} \widehat{\Pi}_{i}^{*}
$$

and

$$
\widehat{\Pi}_{i}^{*}\left(\widehat{N}_{i}+\widehat{\Lambda}_{i}\right)=-\widehat{\Pi}_{i} \widehat{\Pi}_{i}^{*}
$$

Therefore,

$$
\begin{aligned}
& \left(\widehat{\Pi}_{i}+\widehat{N}_{i}+\widehat{\Lambda}_{i}\right)\left(\widehat{\Pi}_{i}+\widehat{N}_{i}+\widehat{\Lambda}_{i}\right)^{*} \\
& \quad=\left(\widehat{N}_{i}+\widehat{\Lambda}_{i}\right)\left(\widehat{N}_{i}+\widehat{\Lambda}_{i}\right)^{*}-\widehat{\Pi}_{i} \widehat{\Pi}_{i}^{*},
\end{aligned}
$$

and using Parseval's identity,

$$
\begin{aligned}
\left\langle\|\boldsymbol{\Pi}+\boldsymbol{N}+\boldsymbol{\Lambda}\|^{2}\right\rangle & =\left\langle(\widehat{\boldsymbol{\Pi}}+\widehat{\boldsymbol{N}}+\widehat{\boldsymbol{\Lambda}}) \cdot(\widehat{\boldsymbol{\Pi}}+\widehat{\boldsymbol{N}}+\widehat{\boldsymbol{\Lambda}})^{*}\right\rangle \\
& =\left\langle(\widehat{\boldsymbol{N}}+\widehat{\boldsymbol{\Lambda}}) \cdot(\widehat{\boldsymbol{N}}+\widehat{\boldsymbol{\Lambda}})^{*}\right\rangle-\left\langle\widehat{\boldsymbol{\Pi}} \cdot \widehat{\boldsymbol{\Pi}}^{*}\right\rangle \\
& =\left\langle\|\boldsymbol{N}+\boldsymbol{\Lambda}\|^{2}\right\rangle-\left\langle\|\boldsymbol{\Pi}\|^{2}\right\rangle .
\end{aligned}
$$

[1] A. Tsinober, An Informal Introduction to Turbulence (Kluwer Academic Publishers, Boston, 2001).

[2] S. B. Pope, Lagrangian PDF methods for turbulent flows, Annu. Rev. Fluid Mech. 26, 23 (1994).

[3] W. Heisenberg, Zur statistischen Theorie der Turbulenz, Zeitschrift für Physik 124, 628 (1948).

[4] A. M. Yaglom, On the acceleration field in a turbulent flow, C. R. Akad. URSS 67(5), 795 (1949).

[5] A. La Porta, G. A. Voth, A. M. Crawford, J. Alexander, and E. Bodenschatz, Fluid particle accelerations in fully developed turbulence, Nature (London) 409, 1017 (2001).

[6] F. Toschi and E. Bodenschatz, Lagrangian properties of particles in turbulence, Annu. Rev. Fluid Mech. 41, 375 (2009).

[7] P. K. Yeung, Lagrangian investigations of turbulence, Annu. Rev. Fluid Mech. 34, 115 (2002).
[8] W. J. T. Bos and R. Rubinstein, On the strength of the nonlinearity in isotropic turbulence, J. Fluid Mech. 733, 158 (2013).

[9] O. Kamps, R. Friedrich, and R. Grauer, Exact relation between Eulerian and Lagrangian velocity increment statistics, Phys. Rev. E 79, 066301 (2009).

[10] H. Homann, O. Kamps, R. Friedrich, and R. Grauer, Bridging from Eulerian to Lagrangian statistics in 3D hydro- and magnetohydrodynamic turbulent flows, New J. Phys. 11, 073020 (2009).

[11] M. Holzer and E. Siggia, Skewed, exponential pressure distributions from Gaussian velocities, Phys. Fluids A 5, 2525 (1993).

[12] K. Yoshimatsu, N. Okamoto, K. Schneider, Y. Kaneda, and M. Farge, Intermittency and scale-dependent statistics in fully developed turbulence, Phys. Rev. E 79, 026303 (2009). 
[13] W. J. T. Bos, B. Kadoch, S. Neffaa, and K. Schneider, Lagrangian dynamics of drift-wave turbulence, Physica D 239, 1269 (2010).

[14] C. Meneveau, Analysis of turbulence in the orthonormal wavelet representation, J. Fluid Mech. 232, 469 (1991).

[15] M. Farge, Wavelet transforms and their applications to turbulence, Annu. Rev. Fluid Mech. 24, 395 (1992).

[16] W. J. T. Bos, L. Liechtenstein, and K. Schneider, Small scale intermittency in anisotropic turbulence, Phys. Rev. E 76, 046310 (2007).

[17] B. Kadoch, W. J. T. Bos, and K. Schneider, Extreme Lagrangian acceleration in confined turbulent flow, Phys. Rev. Lett. 100, 184503 (2008).

[18] F. G. Jacobitz, L. Liechtenstein, K. Schneider, and M. Farge, On the structure and dynamics of sheared and rotating turbulence: Direct numerical simulation and wavelet-based coherent vortex extraction, Phys. Fluids 20, 045103 (2008).

[19] F. G. Jacobitz, K. Schneider, W. J. T. Bos, and M. Farge, On the structure and dynamics of sheared and rotating turbulence: Anisotropy properties and geometrical scale-dependent statistics, Phys. Fluids 22, 085101 (2010).

[20] G. Brethouwer, The effect of rotation on rapidly sheared homogeneous turbulence and passive scalar transport. Linear theory and direct numerical simulation, J. Fluid Mech. 542, 305 (2005).

[21] A. Salhi, F. G. Jacobitz, K. Schneider, and C. Cambon, Nonlinear dynamics and anisotropic structure of rotating sheared turbulence, Phys. Rev. E 89, 013020 (2014).
[22] P. Sagaut and C. Cambon, Homogeneous Turbulence Dynamics (Cambridge University Press, Cambridge, 2008).

[23] R. S. Rogallo, Numerical experiments in homogeneous turbulence, NASA Report No. TM 81315 (1981).

[24] F. G. Jacobitz, S. Sarkar, and C. W. Van Atta, Direct numerical simulations of the turbulence evolution in a uniformly sheared and stably stratified flow, J. Fluid Mech. 342, 231 (1997).

[25] A. Tsinober, P. Vedula, and P. K. Yeung, Random Taylor hypothesis and the behavior of local and convective accelerations in isotropic turbulence, Phys. Fluids 13, 1974 (2001).

[26] H. Tennekes, Eulerian and Lagrangian time microscales in isotropic turbulence, J. Fluid Mech. 67, 561 (1975).

[27] T. Gotoh and D. Fukuyama, Pressure spectrum in homogeneous turbulence, Phys. Rev. Let. 86, 3775 (2001).

[28] J. C. R. Hunt and D. J. Carruthers, Rapid distortion theory and the 'problems' of turbulence, J. Fluid Mech. 212, 497 (1990).

[29] W. J. T. Bos, R. Rubinstein, and L. Fang, Reduction of meansquare advection in turbulent passive scalar mixing, Phys. Fluids 24, 075104 (2012).

[30] T. Ishihara, Y. Kaneda, M. Yokokawa, K. Itakura, and A. Uno, Small-scale statistics in high-resolution direct numerical simulation of turbulence: Reynolds number dependence of one-point velocity gradient statistics, J. Fluid Mech. 592, 335 (2007).

[31] S. Jackson, Research Methods and Statistics: A Critical Thinking Approach (Cengage Learning, Boston, MA, 2015). 